\title{
The Global Analysis of a Stochastic Two-Scale Network Epidemic Dynamic Model with Varying Immunity Period
}

\author{
Divine Wanduku1 ${ }^{*}$, G. S. Ladde ${ }^{2}$ \\ ${ }^{1}$ Department of Mathematical Sciences, Georgia Southern University, Statesboro, USA \\ ${ }^{2}$ Department of Mathematics and Statistics, University of South Florida, Tampa, USA \\ Email: *dwanduku@georgiasouthern.edu, *wandukudivine@yahoo.com, gladde@usf.edu
}

How to cite this paper: Wanduku, D. and Ladde, G.S. (2017) The Global Analysis of a Stochastic Two-Scale Network Epidemic Dynamic Model with Varying Immunity Period. Journal of Applied Mathematics and Physics, 5, 1150-1173.

https://doi.org/10.4236/jamp.2017.55101

Received: April 13, 2017

Accepted: May 23, 2017

Published: May 27, 2017

Copyright $\odot 2017$ by authors and Scientific Research Publishing Inc. This work is licensed under the Creative Commons Attribution International License (CC BY 4.0).

http://creativecommons.org/licenses/by/4.0/

\begin{abstract}
A stochastic SIR epidemic dynamic model with distributed-time-delay, for a two-scale dynamic population is derived. The distributed time delay is the varying naturally acquired immunity period of the removal class of individuals who have recovered from the infection, and have acquired natural immunity to the disease. We investigate the stochastic asymptotic stability of the disease free equilibrium of the epidemic dynamic model, and verify the impact on the eradication of the disease.
\end{abstract}

\section{Keywords}

Disease-Free Steady State, Stochastic Asymptotic Stability, Threshold Value, Positively Self Invariant Set,

Lyapunov Functional Technique

\section{Introduction}

The recent advent of high technology in the area of communication, transportation and basic services, multilateral interactions have afforded efficient global mass flow of human beings, animals, goods, equipments and ideas on the earth's multi-patches surface. As a result of this, the world has become like a neighborhood. Furthermore, the national and binational problems have become the multinational problems. This has generated a sense of cooperation and understanding about the basic needs of human species in the global community. In short, the idea of globalization is spreading in almost all aspects of the human species on the surface of earth. The world today faces the challenge of increasingly high rates of globalization of new human infectious diseases and disease 
strains [1] associated with the high number of inter-patch connections modern efficient global human transportation. For instance, the recent $2009 \mathrm{H} 1 \mathrm{~N} 1 \mathrm{flu}$ pandemic [2] is closely interrelated with the many inter-patch connections facilitated human transportation of the disease. Attempts to study human infectious disease dynamics influenced by human mobility process in complex human meta-population structures are made [3]-[15].

The inclusion of the effects of disease latency or immunity into the epidemic dynamic modeling process leads to more realistic epidemic dynamic models. Furthermore, epidemic dynamic processes in populations exhibiting varying time disease latency or immunity delay periods are represented by differential equation models with distributed time delays. Several studies [16] [17] [18] [19] [20] incorporating distributed delays describing the effects of disease latency or immunity in the dynamics of human infectious diseases have been done. A mathematical SIR (susceptible-infective-removal) epidemic dynamic model with distributed time delays representing the varying time temporal immunity period in the immune population class is studied by Blyuss and Kyrychko [19]. In their study, the existence of positive solution is exhibited. Furthermore, the global asymptotic stability of the disease free and endemic equilibria are shown by using Lyapunov functional technique. Moreover, they presented numerical simulation results for a special case SIR epidemic with temporal immunity. The temporal immunity was represented in the epidemic dynamic model by letting the Dirac delta-function be the integral kernel or the probability density function of the distributed time delay.

Stochastic models also offer a better representation of the reality. Several stochastic dynamic models describing single and multi-group disease dynamics have been investigated [20]-[29]. In [21], a stochastic multi-group SIRS epidemic dynamic models is derived and studied. The random environmental fluctuations manifest as variability in the disease transmission process. In addition, the global positive solution existence is exhibited by the Lyapunov energy function method and a positively self invariant set is defined. Moreover, the the stochastic asymptotic and mean square stability of the disease free equilibrium are exhibited by applying Lyapunov second method. In [22], D. Wanduku and G.S. Ladde derived and studied a stochastic two-scale network constant temporary delayed SIR epidemic model. The temporary immunity period accounts for the time lag during which newly recovered individuals from the disease with conferred infection acquired or natural immunity lose the immunity and regain the susceptible state. They utilized the Lyapunov energy function method to prove the global positive solution process existence, and defined a positively self invariant set. Moreover, the the stochastic asymptotic and mean square stability of the disease free equilibrium are exhibited by applying Lyapunov functional technique. In [20], a stochastic SIR epidemic dynamic model with distributed time delay is studied. Moreover, the stochastic asymptotic stability of the disease free equilibrium is also exhibited by applying the Lyapunov functional techni- 
que.

In this paper we extend the two-scale network SIR temporary delayed epidemic dynamic model [22] into a two-scale network SIR delayed epidemic dynamic model with varying natural immunity period. The varying immunity period accounts for the varying time lengths of immunity within the immune population class. This means that individuals recovering from the disease acquire natural immunity against the disease. Moreover, the immunity period varies for individuals in the immune population class. Furthermore, the acquired immunity wanes with time and the temporary immune individuals are reconverted to the susceptible state.

This work is organized as follows. In Section 2, we derive the distributed time acquired immunity delay epidemic dynamic model. In Section 3, we present the model validation results of the epidemic model. In Section 4, we show the stochastic asymptotic stability of the disease free equilibrium.

\section{Derivation of the SIR Distributed Delay Stochastic Dynamic Model}

In this section, we derive the varying immunity delay effect in the SIR disease dynamics of residents of site $s_{i}^{r}$ in region $C_{r}$ of the two-scale population. We recall the general large scale two level stochastic SIR constant temporary delayed epidemic dynamic model studied is given ([22], (2.7)-(2.9)). We extend the constant temporary immunity effect in [22] into the varying time temporary immunity effect as follows: we assume that for each $r \in I(1, M)$, and $i \in I\left(1, n_{r}\right)$, infectious $\left(I_{i a}^{r u}\right)$ residents of site $s_{i}^{r}$ in region $C_{r}$ visiting site $s_{a}^{u}$ in region $C_{u}$ recover from the disease and acquire natural immunity against the disease immediately after recovery. The recovered individuals further loose immunity and become susceptible to the disease after a period of time $s$, where the immunity period $s$ is an infinite random variable with values between 0 and $\infty$ for the different individuals in the immune population class. Using ideas from [19], we derive and incorporate the varying time acquired immunity delay effect into the epidemic dynamic model ((2.7)-(2.9), [22]) by introducing the term

$$
\rho_{a}^{u} \int_{0}^{\infty} I_{i a}^{r u}(t-s) f_{i a}^{r u}(s) e^{-\delta_{a}^{u} s} \mathrm{~d} s
$$

where $e^{-\delta_{a}^{u} s}$ is the probability that an individual who recovered from disease at an earlier time $t-s$ is still alive at time $t$. Furthermore, $f_{i a}^{r u}(s)$ is the integral kernel [19] representing the probability density of the time $s$ to loose acquired immunity by residents of site $s_{i}^{r}$ in region $C_{r}$ who were previously infectious at their visiting site $s_{a}^{u}$ in region $C_{u}$, and who have recovered from disease acquiring natural immunity with varying time lengths. Moreover, $\int_{0}^{\infty} f_{i a}^{r u}(s) \mathrm{d} s=1$, and $f_{i a}^{r u} \geq 0$. The two level large scale stochastic SIR delayed epidemic dynamic model with varying natural immunity period and which is influenced by the human mobility process [30] is as follows: 


$$
\begin{aligned}
& \mathrm{d} S_{i a}^{r u}=\left\{\begin{array}{l}
{\left[B_{i}^{r}+\sum_{k=1}^{n_{r}} \rho_{i k}^{r r} S_{i k}^{r r}+\sum_{q \neq r}^{M} \sum_{a=1}^{n_{q}} \rho_{i a}^{r q} S_{i a}^{r q}+\varrho_{i}^{r} \int_{0}^{\infty} I_{i i}^{r r}(t-s) f_{i i}^{r r}(s) e^{-\delta_{i}^{r} s} \mathrm{~d} s\right.} \\
\left.-\left(\gamma_{i}^{r}+\sigma_{i}^{r}+\delta_{i}^{r}\right) S_{i i}^{r r}-\sum_{u=1}^{M} \sum_{a=1}^{n_{u}} \beta_{i i a}^{r r u} S_{i i}^{r r} I_{a i}^{r r}\right] \mathrm{d} t \\
-\left[\sum_{u=1}^{M} \sum_{a=1}^{n_{u}} v_{i i a}^{r r u} S_{i i}^{r r} I_{a i}^{u r} \mathrm{~d} w_{i i a}^{r r u}(t)\right], u=r, a=i \\
\sigma_{i j}^{r r} S_{i i}^{r r}+\varrho_{j}^{r} \int_{0}^{\infty} I_{i j}^{r r}(t-s) f_{i j}^{r r}(s) e^{-\delta_{j}^{r s}} \mathrm{~d} s-\left(\rho_{i j}^{r r}+\delta_{j}^{r}\right) S_{i j}^{r r} \\
\left.-\sum_{u=1}^{M} \sum_{a=1}^{n_{u}} \beta_{j i a}^{r r u} S_{i j}^{r r} I_{a j}^{u r}\right] \mathrm{d} t-\left[\sum_{u=1}^{M} \sum_{a=1}^{n_{u}} v_{j i a}^{r u} S_{i j}^{r r} I_{a j}^{u r} \mathrm{~d} w_{j i a}^{r r u}(t)\right], u=r, a=j, j \neq i, \\
{\left[\gamma_{i l}^{r q} S_{i i}^{r r}+\varrho_{l}^{q} \int_{0}^{\infty} I_{i l}^{r q}(t-s) f_{i l}^{r q}(s) \mathrm{e}^{-\delta_{l}^{q} s} \mathrm{~d} s-\left(\rho_{i l}^{r q}+\delta_{l}^{q}\right) S_{i l}^{r q}\right.} \\
\left.-\sum_{u=1}^{M} \sum_{a=1}^{n_{u}} \beta_{l i a}^{q r u} S_{i l}^{r q} I_{a l}^{u q}\right] \mathrm{d} t-\left[\sum_{u=1}^{M} \sum_{a=1}^{n_{u}} v_{l i a}^{q r u} S_{i l}^{r q} I_{a l}^{u q} \mathrm{~d} w_{l i a}^{q r u}(t)\right], u=q, a=l, q \neq r,
\end{array}\right. \\
& \int\left[\sum_{k=1}^{n_{r}} \rho_{i k}^{r r} I_{i k}^{r r}+\sum_{q \neq r}^{M} \sum_{a=1}^{n_{q}} \rho_{i a}^{r q} I_{i a}^{r q}-\varrho_{i}^{r} I_{i i}^{r r}\right. \\
& \left.-\left(\gamma_{i}^{r}+\sigma_{i}^{r}+\delta_{i}^{r}+d_{i}^{r}\right) I_{i i}^{r r}+\sum_{u=1}^{M} \sum_{a=1}^{n_{u}} \beta_{i i a}^{r r u} S_{i i}^{r r} I_{a i}^{u r}\right] \mathrm{d} t \\
& +\left[\sum_{u=1}^{M} \sum_{a=1}^{n_{u}} v_{i i a}^{r r u} S_{i i}^{r r} I_{a i}^{u r} \mathrm{~d} w_{i i a}^{r r u}(t)\right], u=r, a=i \\
& \mathrm{~d} I_{i a}^{r u}=\left\{\left[\sigma_{i j}^{r r} I_{i i}^{r r}-\varrho_{j}^{r} I_{i j}^{r r}-\left(\rho_{i j}^{r r}+\delta_{j}^{r}+d_{j}^{r}\right) I_{i j}^{r r}+\sum_{u=1}^{M} \sum_{a=1}^{n_{u}} \beta_{j i a}^{r u} S_{i j}^{r r} I_{a j}^{u r}\right] \mathrm{d} t\right. \\
& +\left[\sum_{u=1}^{M} \sum_{a=1}^{n_{u}} v_{j i a}^{r r u} S_{i j}^{r r} I_{a j}^{u r} \mathrm{~d} w_{j i a}^{r r u}(t)\right], u=r, a=j, j \neq i, \\
& {\left[\gamma_{i l}^{r q} I_{i i}^{r r}-\varrho_{l}^{q} I_{i l}^{r q}-\left(\rho_{i l}^{r q}+\delta_{l}^{q}+d_{l}^{q}\right) I_{i l}^{r q}+\sum_{u=1}^{M} \sum_{a=1}^{n_{u}} \beta_{l i a}^{q r u} S_{i l}^{r q} I_{a l}^{u q}\right] \mathrm{d} t} \\
& +\left[\sum_{u=1}^{M} \sum_{a=1}^{n_{u}} v_{l i a}^{q r u} S_{i l}^{r q} I_{a l}^{u q} \mathrm{~d} w_{l i a}^{q r u}(t)\right], u=q, a=l, q \neq r, \\
& R_{i a}^{r u}=\left\{\begin{array}{l}
{\left[\sum_{k=1}^{n_{r}} \rho_{i k}^{r r} R_{i k}^{r r}+\sum_{q \neq r}^{M} \sum_{a=1}^{n_{q}} \rho_{i l}^{r q} R_{i l}^{r q}+\varrho_{i}^{r} I_{i i}^{r r}-\varrho_{i}^{r} \int_{0}^{\infty} I_{i i}^{r r}(t-s) f_{i i}^{r r}(s) e^{-\delta_{i}^{r} s} \mathrm{~d} s\right.} \\
\left.-\left(\gamma_{i}^{r}+\sigma_{i}^{r}+\delta_{i}^{r}\right) R_{i i}^{r r}\right] \mathrm{d} t, u=r, a=i \\
{\left[\begin{array}{l}
\sigma_{i j}^{r r} R_{i i}^{r r}+\varrho_{j}^{r} I_{i j}^{r r}-\varrho_{j}^{r} \int_{0}^{\infty} I_{i j}^{r r}(t-s) f_{i j}^{r r}(s) e^{-\delta_{j}^{r} s} \mathrm{~d} s \\
\left.-\left(\rho_{i j}^{r r}+\delta_{j}^{r}\right) R_{i j}^{r r}\right] \mathrm{d} t, u=r, a=j, j \neq i, \\
{\left[\gamma_{i l}^{r q} R_{i i}^{r r}+\varrho_{l}^{q} I_{i l}^{r q}-\varrho_{l}^{q} \int_{0}^{\infty} I_{i l}^{r q}(t-s) f_{i l}^{r q}(s) e^{-\delta_{l}^{q} s} \mathrm{~d} s\right.} \\
\left.-\left(\rho_{i l}^{r q}+\delta_{l}^{q}\right) R_{i l}^{r q}\right] \mathrm{d} t, u=q, a=l, q \neq r,
\end{array}\right.}
\end{array}\right.
\end{aligned}
$$

where all parameters are previously defined. Furthermore, for each $r \in I(1, M)$, and $i \in I\left(1, n_{r}\right)$, we have the following initial conditions

$$
\begin{aligned}
& \left(S_{i a}^{r u}(t), I_{i a}^{r u}(t), R_{i a}^{r u}(t)\right)=\left(\varphi_{i a 1}^{r u}(t), \varphi_{i a 2}^{r u}(t), \varphi_{i a 3}^{r u}(t)\right), t \in\left[-\infty, t_{0}\right], \\
& \varphi_{i a k}^{r u} \in \mathcal{C}\left(\left[-\infty, t_{0}\right], \mathbb{R}_{+}\right), \forall k=1,2,3, \forall r, q \in I(1, M), a \in I\left(1, n_{u}\right), i \in I\left(1, n_{r}\right), \\
& \varphi_{i a k}^{r u}\left(t_{0}\right)>0, \forall k=1,2,3,
\end{aligned}
$$

where $\mathcal{C}\left(\left[-\infty, t_{0}\right], \mathbb{R}_{+}\right)$is the space of continuous functions with the supremum norm

$$
\|\varphi\|_{\infty}=\operatorname{Sup}_{-\infty \leq t \leq t_{0}}|\varphi(t)| .
$$

and $w$ is a Wierner process. Furthermore, the random continuous functions $\varphi_{i a k}^{r u}, k=1,2,3$ are $\digamma_{0}$-measurable, or independent of $w(t)$ for all $t \geq t_{0}$. 
We express the state of system (2.1)-(2.3) in vector form and use it, subsequently. We denote

$$
\begin{aligned}
& x_{i a}^{r u}=\left(S_{i a}^{r u}, I_{i a}^{r u}, R_{i a}^{r u}\right)^{\mathrm{T}} \in \mathbb{R}^{3} \\
& x_{i 0}^{r u}=\left(x_{i 1}^{r u T}, x_{i 2}^{r u T}, \cdots, x_{i, n u}^{r u T}\right)^{\mathrm{T}} \in \mathbb{R}^{3 n_{u}}, \\
& x_{00}^{r u}=\left(x_{10}^{r u T}, x_{20}^{r u T}, \cdots, x_{n_{r} 0}^{r u T}\right)^{\mathrm{T}} \in \mathbb{R}^{3 n_{r} n_{u}}, \\
& x_{00}^{r 0}=\left(x_{00}^{r 1 T}, x_{00}^{r 2 T}, \cdots, x_{00}^{r M T}\right)^{\mathrm{T}} \in \mathbb{R}^{3 n_{r} \sum_{u=1}^{M} n_{u}}, \\
& x_{00}^{00}=\left(x_{00}^{10}, x_{00}^{20}, \cdots, x_{00}^{M 0}\right)^{\mathrm{T}} \in \mathbb{R}^{3\left(\sum_{r=1}^{M} n_{r}\right)\left(\sum_{u=1}^{M} n_{u}\right)},
\end{aligned}
$$

where $r, u \in I(1, M), \quad i \in I\left(1, n_{r}\right), \quad a \in I_{i}^{r}\left(1, n_{u}\right)$. We set $n=\sum_{u=1}^{M} n_{u}$.

Definition 2.1.

1. $\mathrm{p}$-norm in $\mathbb{R}^{3 n^{2}}:$ Let $z_{00}^{00} \in \mathbb{R}^{3 n^{2}}$ be an arbitrary vector defined in (2.6), where $\quad z_{i a}^{r u}=\left(z_{i a 1}^{r u 0}, z_{i a 2}^{r u 0}, z_{i a 3}^{r u 0}\right)^{\mathrm{T}} \quad$ whenever $\quad r, u \in I(1, M), \quad i \in I\left(1, n_{r}\right)$, $a \in I_{i}^{r}\left(1, n_{u}\right)$. The p-norm on $\mathbb{R}^{3 n^{2}}$ is defined as follows

$$
\left\|z_{00}^{00}\right\|_{p}=\left(\sum_{r=1}^{M} \sum_{u=1}^{M} \sum_{i=1}^{n_{r}} \sum_{a=1}^{n_{u}} \sum_{j=1}^{3}\left|z_{i a j}^{r u 0}\right|^{p}\right)^{\frac{1}{p}}
$$

whenever $1 \leq p<\infty$, and

$$
\bar{Z} \equiv\left\|z_{00}^{00}\right\|_{p}=\max _{1 \leq r, u \leq M, 1 \leq i \leq n_{r}, 1 \leq a \leq n_{u}, 1 \leq j \leq 3}\left|z_{i a j}^{r u 0}\right|,
$$

whenever $p=\infty$. Let

$$
\underline{k} \equiv k_{00 \min }^{00}=\min _{1 \leq r, u \leq M, 1 \leq i \leq n_{r}, 1 \leq a \leq n_{u}}\left|k_{i a}^{r u}\right| .
$$

2. Closed Ball in $\mathbb{R}^{3 n^{2}}$ : Let $z_{00}^{* 00} \in \mathbb{R}^{3 n^{2}}$ be fixed. The closed ball in $\mathbb{R}^{3 n^{2}}$ with center at $z_{00}^{* 00}$ and radius $r>0$ denoted $\overline{\mathfrak{B}}_{\mathbb{R}^{3 n^{2}}}\left(z_{00}^{* 00} ; r\right)$ is the set

$$
\overline{\mathfrak{B}}_{\mathbb{R}^{3 n^{2}}}\left(z_{00}^{* 00} ; r\right)=\left\{z_{00}^{00} \in \mathbb{R}^{3 n^{2}}:\left\|z_{00}^{00}-z_{00}^{* 00}\right\|_{p} \leq r\right\}
$$

In addition, from (2.1)-(2.3), define the vector $y_{00}^{00} \in \mathbb{R}^{n^{2}}$ as follows: For $i \in I\left(1, n_{r}\right), \quad l \in I_{i}^{r}\left(1, n_{q}\right), \quad r \in I(1, M)$ and $q \in I^{r}(1, M)$,

$$
\begin{aligned}
& y_{i a}^{r u}=S_{i a}^{r u}+I_{i a}^{r u}+R_{i a}^{r u} \in \mathbb{R}_{+}=[0, \infty) \\
& y_{i 0}^{r u}=\left(y_{i 1}^{r u}, y_{i 2}^{r u}, \cdots, y_{i, n u}^{r u}\right)^{\mathrm{T}} \in \mathbb{R}_{+}^{n_{u}}, \\
& y_{00}^{r u}=\left(y_{10}^{r u T}, y_{20}^{r u T}, \ldots, y_{n_{r} 0}^{r u T}\right)^{\mathrm{T}} \in \mathbb{R}_{+}^{n_{r} n_{u}}, \\
& y_{00}^{r 0}=\left(y_{00}^{r 1 T}, y_{00}^{r 2 T}, \cdots, y_{00}^{r M T}\right)^{\mathrm{T}} \in \mathbb{R}_{+}^{n_{r} \sum_{u=1}^{M} n_{u}}, \\
& y_{00}^{00}=\left(y_{00}^{10 T}, y_{00}^{20 T}, \cdots, y_{00}^{M 0 T}\right)^{\mathrm{T}} \in \mathbb{R}_{+}^{\left(\sum_{r=1}^{M} n_{r}\right)\left(\sum_{u=1}^{M} n_{u}\right)},
\end{aligned}
$$

and obtain

$$
\mathrm{d} y_{i l}^{r q}=\left\{\begin{array}{l}
{\left[B_{i}^{r}+\sum_{k \neq i}^{n_{r}} \rho_{i k}^{r r} y_{i k}^{r r}+\sum_{q \neq r}^{M} \sum_{a=1}^{n_{q}} \rho_{i a}^{r q} y_{i a}^{r q}-\left(\gamma_{i}^{r}+\sigma_{i}^{r}+\delta_{i}^{r}\right) y_{i i}^{r r}-d_{i}^{r} I_{i i}^{r r}\right] \mathrm{d} t, \text { for } q=r, l=i} \\
{\left[\sigma_{i j}^{r r} y_{i i}^{r r}-\left(\rho_{i j}^{r r}+\delta_{j}^{r}\right) y_{i j}^{r r}-d_{j}^{r} I_{i j}^{r r}\right] \mathrm{d} t, \text { for } q=r, a=j \text { and } i \neq j,} \\
\left.\left[\gamma_{i l}^{r q} y_{i i}^{r r}-\left(\rho_{i l}^{r q}+\delta_{l}^{q}\right)\right) y_{i l}^{r q}-d_{l}^{q} I_{i l}^{r q}\right] \mathrm{d} t, \text { for } q \neq r, y_{i l}^{r q}\left(t_{0}\right) \geq 0,
\end{array}\right.
$$




\section{Model Validation Results}

In the following we state and prove a positive solution process existence theorem for the delayed system (2.1)-(2.3). We utilize the Lyapunov energy function method in our earlier study [22] to establish the results of this theorem. We observe from (2.1)-(2.3) that (2.3) decouples from the first two equations in the system. Therefore, it suffices to prove the existence of positive solution process for $\left(S_{i a}^{r u}, I_{i a}^{r u}\right)$. We utilize the notations (2.6) and keep in mind that $X_{i a}^{r u}=\left(S_{i a}^{r u}, I_{i a}^{r u}\right)^{\mathrm{T}}$.

Theorem 3.1. Let $r, u \in I(1, M), i \in I\left(1, n_{r}\right)$ and $a \in I\left(1, n_{u}\right)$. Given any initial conditions (2.4) and (2.5), there exists a unique solution process $X_{i a}^{r u}(t, w)=\left(S_{i a}^{r u}(t, w), I_{i a}^{r u}(t, w)\right)^{\mathrm{T}}$ satisfying (2.1) and (2.2), for all $t \geq t_{0}$. Moreover, the solution process is positive for all $t \geq t_{0}$ a.s. That is,

$$
S_{i a}^{r u}(t, w)>0, I_{i a(t, w)}^{r u}>0, \forall t \geq t_{0} \text { a.s. }
$$

Proof:

It is easy to see that the coefficients of (2.1) and (2.2) satisfy the local Lipschitz condition for the given initial data (2.4). Therefore there exist a unique maximal local solution $X_{i a}^{r u}(t, w)$ on $t \in\left[-\infty, \tau_{e}(w)\right]$, where $\tau_{e}(w)$ is the first hitting time or the explosion time [31]. We show subsequently that $S_{i a}^{r u}(t, w), I_{i a}^{r u}(t, w)>0$ for all $t \in\left[-\infty, \tau_{e}(w)\right]$ almost surely. We define the following stopping time

$$
\left\{\begin{array}{l}
\tau_{+}=\sup \left\{t \in\left(t_{0}, \tau_{e}(w)\right):\left.S_{i a}^{r u}\right|_{\left[t_{0}, t\right]}>0 \text { and }\left.I_{i a}^{r u}\right|_{\left[t_{0}, t\right]}>0\right\}, \\
\tau_{+}(t)=\min \left(t, \tau_{+}\right), \text {for } t \geq t_{0} .
\end{array}\right.
$$

and we show that $\tau_{+}(t)=\tau_{e}(w)$ a.s. Suppose on the contrary that $P\left(\tau_{+}(t)<\tau_{e}(w)\right)>0$. Let $w \in\left\{\tau_{+}(t)<\tau_{e}(w)\right\}$, and $t \in\left[t_{0}, \tau_{+}(t)\right)$. Define

$$
\left\{\begin{array}{l}
V\left(X_{00}^{00}\right)=\sum_{r=1}^{n_{M}} \sum_{i=1}^{n_{r}} \sum_{u=1}^{M} \sum_{a=1}^{n_{u}} V\left(X_{i a}^{r u}\right), \\
V\left(X_{i a}^{r u}\right)=\ln \left(S_{i a}^{r u}\right)+\ln \left(I_{i a}^{r u}\right), \forall t \leq \tau_{+}(t) .
\end{array}\right.
$$

We rewrite (3.2) as follows

$$
V\left(X_{00}^{00}\right)=\sum_{r=1}^{M} \sum_{i=1}^{n_{r}}\left[V\left(X_{i i}^{r r}\right)+\sum_{j \neq i}^{n_{r}} V\left(X_{i j}^{r r}\right)+\sum_{q \neq r}^{M} \sum_{l=1}^{n_{q}} V\left(X_{i l}^{r q}\right)\right],
$$

And (3.3) further implies that

$$
\mathrm{d} V\left(X_{00}^{00}\right)=\sum_{r=1}^{M} \sum_{i=1}^{n_{r}}\left[\mathrm{~d} V\left(X_{i i}^{r r}\right)+\sum_{j \neq i}^{n_{r}} \mathrm{~d} V\left(X_{i j}^{r r}\right)+\sum_{q \neq r}^{M} \sum_{l=1}^{n_{q}} \mathrm{~d} V\left(X_{i l}^{r q}\right)\right],
$$

where $\mathrm{d} V$ is the Ito-Doob differential operator with respect to the system (2.1)-(2.3). We express the terms on the right-hand-side of (3.4) in the following:

Site Level: From (3.2) the terms on the right-hand-side of (3.4) for the case of $u=r, a=i$ 


$$
\begin{aligned}
\mathrm{d} V\left(X_{i i}^{r r}\right)= & {\left[\frac{B_{i}^{r}}{S_{i i}^{r r}}+\sum_{k \neq i}^{n_{r}} \rho_{i k}^{r r} \frac{S_{i k}^{r r}}{S_{i i}^{r r}}+\sum_{q \neq r}^{M} \sum_{l=1}^{n_{q}} \rho_{i a}^{r q} \frac{S_{i a}^{r q}}{S_{i i}^{r r}}+\frac{\rho_{i}^{r}}{S_{i i}^{r r}} \int_{0}^{\infty} I_{i i}^{r r}(t-s) f_{i i}^{r r}(s) e^{-\delta_{i}^{r} s} \mathrm{~d} s\right.} \\
& \left.-\left(\gamma_{i}^{r}+\sigma_{i}^{r}+\delta_{i}^{r}\right)-\sum_{u=1}^{M} \sum_{a=1}^{n_{u}} \beta_{i i a}^{r r u} I_{a i}^{u r}-\frac{1}{2} \sum_{u=1 a=1}^{M} \sum^{n_{u}}\left(v_{i i a}^{r r u}\right)^{2}\left(I_{a i}^{u r}\right)^{2}\right] \mathrm{d} t \\
& +\left[\sum_{k \neq i}^{n_{r}} \rho_{i k}^{r r} \frac{I_{i k}^{r r}}{S_{i i}^{r r}}+\sum_{q \neq r}^{M} \sum_{l=1}^{n_{q}} \rho_{i a}^{r q} \frac{I_{i a}^{r q}}{S_{i i}^{r r}}-\varrho_{i}^{r}-\left(\gamma_{i}^{r}+\sigma_{i}^{r}+\delta_{i}^{r}+d_{i}^{r}\right)\right. \\
& \left.-\sum_{u=1 a=1}^{M} \sum_{u}^{n_{u}} \beta_{i i a}^{r u} \frac{S_{i i}^{r r}}{I_{i i}^{r r}} I_{a i}^{u r}-\frac{1}{2} \sum_{u=1 a=1}^{M} \sum_{u}^{n_{u}}\left(v_{i i a}^{r r}\right)^{2} \frac{\left(S_{i i}^{r r}\right)^{2}}{\left(I_{i i}^{r r}\right)^{2}}\left(I_{a i}^{u r}\right)^{2}\right] \mathrm{d} t \\
& -\sum_{u=1}^{M} \sum_{a=1}^{n_{u}} v_{i i a}^{r u} I_{a i}^{u r} \mathrm{~d} w_{i i a}^{r r u}(t)+\sum_{u=1}^{M} \sum_{a=1}^{n_{u}} v_{i i a}^{r u} \frac{S_{i i}^{r r}}{I_{i i}^{r r}} I_{a i}^{u r} \mathrm{~d} w_{i i a}^{r r u}(t)
\end{aligned}
$$

Intra-regional Level: From (3.2) the terms on the right-hand-side of (3.4) for the case of $u=r, a=j, j \neq i$

$$
\begin{aligned}
d V\left(X_{i j}^{r r}\right)= & {\left[\sigma_{i j}^{r r} \frac{S_{i i}^{r r}}{S_{i j}^{r r}}+\frac{\varrho_{j}^{r}}{S_{i j}^{r r}} \int_{0}^{\infty} I_{i j}^{r r}(t-s) f_{i j}^{r r}(s) e^{-\delta_{j}^{r} s} \mathrm{~d} s\right.} \\
& \left.-\left(\rho_{i j}^{r r}+\delta_{j}^{r}\right)-\sum_{u=1 a=1}^{M} \sum_{j u}^{n_{u}} \beta_{j i a}^{r r u} I_{a j}^{u r}-\frac{1}{2} \sum_{u=1}^{M} \sum_{a=1}^{n_{u}}\left(v_{j i a}^{r r u}\right)^{2}\left(I_{a j}^{u r}\right)^{2}\right] \mathrm{d} t \\
& +\left[\sigma_{i j}^{r r} \frac{I_{i i}^{r r}}{I_{i j}^{r r}}-\varrho_{j}^{r}-\left(\rho_{i j}^{r r}+\delta_{j}^{r}+d_{j}^{r}\right)\right. \\
& \left.+\sum_{u=1 a=1}^{M} \sum_{j u}^{n_{u}} \beta_{j i a}^{r r u} \frac{S_{i j}^{r r}}{I_{i j}^{r r}} I_{a j}^{u r}-\frac{1}{2} \sum_{u=1}^{M} \sum_{a=1}^{n_{u}}\left(v_{j i a}^{r r u}\right)^{2} \frac{\left(S_{i j}^{r r}\right)^{2}}{\left(I_{i j}^{r r}\right)^{2}}\left(I_{a j}^{u r}\right)^{2}\right] \mathrm{d} t \\
& -\sum_{u=1}^{M} \sum_{a=1}^{n_{u}} v_{j i a}^{r r u} I_{a j}^{u r} \mathrm{~d} w_{j i a}^{r r u}(t)+\sum_{u=1}^{M} \sum_{a=1}^{n_{u}} v_{j i a}^{r r u} \frac{S_{i j}^{r r}}{I_{i j}^{r r}} I_{a j}^{u r} \mathrm{~d} w_{j i a}^{r r u}(t)
\end{aligned}
$$

Regional Level: From (3.2) the terms on the right-hand-side of (3.4) for the case of $u=q, q \neq r, a=l$,

$$
\begin{aligned}
& \mathrm{d} V\left(X_{i l}^{r q}\right)=\left[\gamma_{i l}^{r q} \frac{S_{i i}^{r r}}{S_{i q}^{r q}}+\frac{\varrho_{l}^{q}}{S_{i l}^{r q}} \int_{0}^{\infty} I_{i l}^{r q}(t-s) f_{i l}^{r q}(s) e^{-\delta_{l}^{q} s} \mathrm{~d} s\right. \\
& \left.-\left(\rho_{i l}^{r q}+\delta_{l}^{q}\right)-\sum_{u=1 a=1}^{M} \sum_{\text {lia }}^{n_{u}} \beta_{a l}^{\text {qru }} I_{a}^{u q}-\frac{1}{2} \sum_{u=1 a=1}^{M} \sum^{n_{u}}\left(v_{\text {lia }}^{\text {qru }}\right)^{2}\left(I_{a l}^{u q}\right)^{2}\right] \mathrm{d} t \\
& +\left[\gamma_{i l}^{r q} \frac{I_{i i}^{r r}}{I_{i l}^{r q}}-\varrho_{l}^{q}-\left(\rho_{i l}^{r q}+\delta_{l}^{q}+d_{l}^{q}\right)\right. \\
& +\sum_{u=1 a=1}^{M} \sum_{\text {lia }}^{n_{u}} \beta_{i l}^{q r u} \frac{S_{i l}^{r q}}{I_{i l}^{r q}} I_{a l}^{u q}-\frac{1}{2} \sum_{u=1 a=1}^{M} \sum_{n_{u}}^{n_{l i a}}\left(v_{\text {lia }}^{q r u} \frac{\left(S_{i l}^{r q}\right)^{2}}{\left(I_{i l}^{r q}\right)^{2}}\left(I_{a l}^{u q}\right)^{2}\right] \mathrm{d} t \\
& -\sum_{u=1}^{M} \sum_{a=1}^{n_{u}} v_{\text {lia }}^{q r u} I_{a l}^{u q} d w_{\text {lia }}^{q r u}(t)+\sum_{u=1}^{M} \sum_{a=1}^{n_{u}} v_{\text {lia }}^{q r u} \frac{S_{i l}^{r q}}{I_{i l}^{r q}} I_{a l}^{u q} \mathrm{~d} w_{\text {lia }}^{q r u}(t)
\end{aligned}
$$

It follows from (3.5)-(3.7), (3.4), and (3.1) that for $t<\tau_{+}(t)$, 


$$
\begin{aligned}
& V\left(X_{00}^{00}(t)\right)-V\left(X_{00}^{00}\left(t_{0}\right)\right) \\
& \geq \sum_{r=1}^{M} \sum_{i=1}^{n_{r}} \int_{t_{0}}^{t}\left[\frac{\varrho_{i}^{r}}{S_{i i}^{r r}} \int_{0}^{\infty} I_{i i}^{r r}(t-s) f_{i i}^{r r}(s) e^{-\delta_{i}^{r} s} \mathrm{~d} s-\left(\gamma_{i}^{r}+\sigma_{i}^{r}+\delta_{i}^{r}\right)\right. \\
& \left.-\sum_{u=1}^{M} \sum_{a=1}^{n_{u}} \beta_{i i a}^{r r u} I_{a i}^{u r}-\frac{1}{2} \sum_{u=1}^{M} \sum_{a=1}^{n_{u}}\left(v_{i i a}^{r r u}\right)^{2}\left(I_{a i}^{u r}\right)^{2}\right] \mathrm{d} s+\sum_{r=1}^{M} \sum_{i=1}^{n_{r}} \int_{t_{0}}^{t}\left[-\varrho_{i}^{r}-\left(\gamma_{i}^{r}+\sigma_{i}^{r}+\delta_{i}^{r}+d_{i}^{r}\right)\right. \\
& \left.-\sum_{u=1}^{M} \sum_{a=1}^{n_{u}} \beta_{i i a}^{r r u} \frac{S_{i i}^{r r}}{I_{i i}^{r r}} I_{a i}^{u r}-\frac{1}{2} \sum_{u=1}^{M} \sum_{a=1}^{n_{u}}\left(v_{i i a}^{r r u}\right)^{2} \frac{\left(S_{i i}^{r r}\right)^{2}}{\left(I_{i i}^{r r}\right)^{2}}\left(I_{a i}^{u r}\right)^{2}\right] \mathrm{d} s-\sum_{r=1}^{M} \sum_{i=1}^{n_{r}} \sum_{u=1}^{M} \sum_{a=1}^{n_{u}} \int_{t_{0}}^{t} v_{i i a}^{r r u} I_{a i}^{u r} \mathrm{~d} w_{i i a}^{r r u}(s) \\
& +\sum_{r=1}^{M} \sum_{i=1}^{n_{r}} \sum_{u=1}^{M} \sum_{a=1}^{n_{u}} \int_{t_{0}}^{t} V_{i i a}^{r r u} \frac{S_{i i}^{r r}}{I_{i i}^{r r}} I_{a i}^{u r} \mathrm{~d} w_{i i a}^{r r u}(s)+\sum_{r=1}^{M} \sum_{i=1}^{n_{r}} \sum_{j \neq i}^{n_{r}} \int_{t_{0}}^{t}\left[\frac{\varrho_{j}^{r}}{S_{i j}^{r r}} \int_{0}^{\infty} I_{i j}^{r r}(t-s) f_{i j}^{r r}(s) e^{-\delta_{j}^{r} s} \mathrm{~d} s\right. \\
& \left.-\left(\rho_{i j}^{r r}+\delta_{j}^{r}\right)-\sum_{u=1}^{M} \sum_{a=1}^{n_{u}} \beta_{j i a}^{r u} u_{a j}^{u r}-\frac{1}{2} \sum_{u=1}^{M} \sum_{a=1}^{n_{u}}\left(v_{j i a}^{r r u}\right)^{2}\left(I_{a j}^{u r}\right)^{2}\right] \mathrm{d} s \\
& +\sum_{r=1}^{M} \sum_{i=1}^{n_{r}} \sum_{j \neq i}^{n_{r}} \int_{t_{0}}^{t}\left[-\varrho_{j}^{r}-\left(\rho_{i j}^{r r}+\delta_{j}^{r}+d_{j}^{r}\right)-\frac{1}{2} \sum_{u=1}^{M} \sum_{a=1}^{n_{u}}\left(v_{j i a}^{r r u}\right)^{2} \frac{\left(S_{i j}^{r r}\right)^{2}}{\left(I_{i j}^{r r}\right)^{2}}\left(I_{a j}^{u r}\right)^{2}\right] \mathrm{d} s \\
& -\sum_{r=1}^{M} \sum_{i=1}^{n_{r}} \sum_{j \neq i}^{n_{r}} \sum_{u=1}^{M} \sum_{a=1}^{n_{u}} \int_{t_{0}}^{t} v_{j i a}^{r r u} I_{a j}^{u r} \mathrm{~d} w_{j i a}^{r r u}(s)+\sum_{r=1}^{M} \sum_{i=1}^{n_{r}} \sum_{j \neq i}^{n_{r}} \sum_{u=1}^{M} \sum_{a=1}^{n_{u}} \int_{t_{0}}^{t} v_{j i a}^{r r u} \frac{S_{i j}^{r r}}{I_{i j}^{r r}} I_{a j}^{u r} \mathrm{~d} w_{j i a}^{r r u}(s)
\end{aligned}
$$

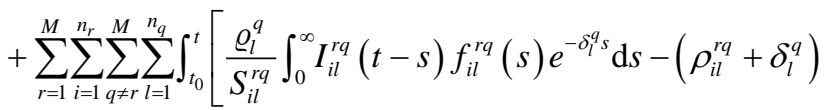

$$
\begin{aligned}
& \left.-\sum_{u=1}^{M} \sum_{a=1}^{n_{u}} \beta_{\text {lia }}^{q r u} I_{a l}^{u q}-\frac{1}{2} \sum_{u=1}^{M} \sum_{a=1}^{n_{u}}\left(v_{\text {lia }}^{q r u}\right)^{2}\left(I_{a l}^{u q}\right)^{2}\right] \mathrm{d} s+\sum_{r=1}^{M} \sum_{i=1}^{n_{r}} \sum_{q \neq r}^{M} \sum_{l=1}^{n_{q}} \int_{t_{0}}^{t}\left[-\varrho_{l}^{q}-\left(\rho_{i l}^{r q}+\delta_{l}^{q}+d_{l}^{q}\right)\right. \\
& \left.-\frac{1}{2} \sum_{u=1}^{M} \sum_{a=1}^{n_{u}}\left(v_{l i a}^{q r u}\right)^{2} \frac{\left(S_{i l}^{r q}\right)^{2}}{\left(I_{i l}^{r q}\right)^{2}}\left(I_{a l}^{u q}\right)^{2}\right] \mathrm{ds}-\sum_{r=1}^{M} \sum_{i=1}^{n_{r}} \sum_{q \neq r}^{M} \sum_{l=1}^{n_{q}} \sum_{u=1}^{M} \sum_{a=1}^{n_{u}} \int_{t_{0}}^{t} v_{l i a}^{q r u} I_{a l}^{u q} \mathrm{~d} w_{l i a}^{q r u}(s) \\
& +\sum_{r=1}^{M} \sum_{i=1}^{n_{r}} \sum_{q \neq r}^{M} \sum_{l=1}^{n_{q}} \sum_{u=1}^{M} \sum_{a=1}^{n_{u}} \int_{t_{0}}^{t} t_{l i a}^{q r u} \frac{S_{i l}^{r q}}{I_{i l}^{r q}} I_{a l}^{u q} \mathrm{~d} w_{l i a}^{q r u}(s)
\end{aligned}
$$

Taking the limit on (3.8) as $t \rightarrow \tau_{+}(t)$, it follows from (3.2) and (3.1) that the left-hand-side $V\left(X_{00}^{00}(t)\right)-V\left(X_{00}^{00}\left(t_{0}\right)\right) \leq-\infty \quad$ (since from (3.2) and (3.1), $\left.V\left(X_{i a}^{r u}\left(\tau_{+}(t)\right)\right)=\ln S_{i a}^{r u}\left(\tau_{+}(t)\right)+\ln I_{i a}^{r u}\left(\tau_{+}(t)\right)=-\infty\right)$. This contradicts the finiteness of the right-hand-side of the inequality (3.8). Hence $\tau_{+}(t)=\tau_{e}(w)$ a.s. We show subsequently that $\tau_{e}(w)=\infty$.

Let $k>0$ be a positive integer such that $\left\|\varphi_{00}^{00}\right\|_{1} \leq k$, where the vector of initial values $\varphi_{00}^{00}=\left(\varphi_{i a}^{r u}\right)_{1 \leq r, u \leq M, 1 \leq i \leq n_{r}, 1 \leq a \leq n_{u}} \in \mathbb{R}^{2 n^{2}}$ is defined in (2.4). Furthermore, $\|\cdot\|_{1}$ is the $p$-sum norm (2.7) for the case of $p=1$. We define the stopping time

$$
\left\{\begin{array}{l}
\tau_{k}=\sup \left\{t \in\left[t_{0}, \tau_{e}\right):\left\|X_{00}^{00}(s)\right\|_{1} \leq k, s \in[0, t]\right\} \\
\tau_{k}(t)=\min \left(t, \tau_{k}\right) .
\end{array}\right.
$$

where from (2.7),

$$
\left\|X_{00}^{00}(s)\right\|_{1}=\sum_{r=1}^{M} \sum_{u=1}^{M} \sum_{i=1}^{n_{r}} \sum_{a=1}^{n_{u}}\left(S_{i a}^{r u}(s)+I_{i a}^{r u}(s)\right) .
$$

It is easy to see that as $k \rightarrow \infty, \tau_{k}$ increases. Set $\lim _{k \rightarrow \infty} \tau_{k}(t)=\tau_{\infty}$. Then 
$\tau_{\infty} \leq \tau_{e}$ a.s. We show in the following that: (1) $\tau_{e}=\tau_{\infty}$ a.s. $\Leftrightarrow P\left(\tau_{e} \neq \tau_{\infty}\right)=0$, (2) $\tau_{\infty}=\infty$ a.s. $\Leftrightarrow P\left(\tau_{\infty}=\infty\right)=1$.

Suppose on the contrary that $P\left(\tau_{\infty}<\tau_{e}\right)>0$. Let $w \in\left\{\tau_{\infty}<\tau_{e}\right\}$ and $t \leq \tau_{\infty}$. In the same structure form as (3.2) and (3.4), define

$$
\left\{\begin{array}{l}
V_{1}\left(X_{00}^{00}\right)=\sum_{r=1}^{M} \sum_{i=1}^{n_{r}} \sum_{u=1}^{M} \sum_{a=1}^{n_{u}} V\left(X_{i a}^{r u}\right), \\
V_{1}\left(X_{i a}^{r u}\right)=e^{\delta_{a}^{u} t}\left(S_{i a}^{r u}+I_{i a}^{r u}\right), \forall t \leq \tau_{k}(t) .
\end{array}\right.
$$

From (3.11), using the expression (3.4), the Ito-Doob differential $\mathrm{d} V_{1}$ with respect to the system (2.1)-(2.3) is given as follows:

Site Level: From (3.11), the terms of the right-hand-side of (3.4) for the case of $u=r, a=i$

$$
\begin{aligned}
\mathrm{d} V_{1}\left(X_{i i}^{r r}\right)= & e^{\delta_{i}^{r} t}\left[B_{i}^{r}+\sum_{k \neq i}^{n_{r}} \rho_{i k}^{r r} S_{i k}^{r r}+\sum_{q \neq r}^{M} \sum_{l=1}^{n_{q}} \rho_{i a}^{r q} S_{i a}^{r q}\right. \\
& \left.+\varrho_{i}^{r} \int_{0}^{\infty} I_{i i}^{r r}(t-s) f_{i i}^{r r}(s) e^{-\delta_{i}^{r} s} \mathrm{~d} s-\left(\gamma_{i}^{r}+\sigma_{i}^{r}\right) S_{i i}^{r r}\right] \mathrm{d} t \\
& +e^{\delta_{i}^{r} t}\left[\sum_{k \neq i}^{n_{r}} \rho_{i k}^{r r} I_{i k}^{r r}+\sum_{q \neq r}^{M} \sum_{l=1}^{n_{q}} \rho_{i a}^{r q} I_{i a}^{r q}-\varrho_{i}^{r} I_{i i}^{r r}-\left(\gamma_{i}^{r}+\sigma_{i}^{r}+d_{i}^{r}\right) I_{i i}^{r r}\right] \mathrm{d} t
\end{aligned}
$$

Intra-regional Level: From (3.11), the terms of the right-hand-side of (3.4) for the case of $u=r, a=j, j \neq i$

$$
\begin{aligned}
\mathrm{d} V_{1}\left(X_{i j}^{r r}\right)= & \mathrm{e}^{\delta_{i}^{r} t}\left[\sigma_{i j}^{r r} S_{i i}^{r r}+\varrho_{j}^{r} \int_{0}^{\infty} I_{i j}^{r r}(t-s) f_{i j}^{r r}(s) e^{-\delta_{j}^{r} s} \mathrm{~d} s-\rho_{i j}^{r r} S_{i j}^{r r}\right] \mathrm{d} t \\
& +e^{\delta_{j}^{r} t}\left[\sigma_{i j}^{r r} I_{i i}^{r r}+\varrho_{j}^{r} I_{i j}^{r r}-\left(\rho_{i j}^{r r}+d_{j}^{r}\right) I_{i j}^{r r}\right] \mathrm{d} t
\end{aligned}
$$

Regional Level: From (3.11), the terms of the right-hand-side of (3.4) for the case of $u=q, q \neq r, a=l$

$$
\begin{aligned}
\mathrm{d} V_{1}\left(X_{i l}^{r q}\right)= & e^{\delta_{l}^{q} t}\left[\gamma_{i l}^{r q} S_{i i}^{r r}+\varrho_{l}^{q} \int_{0}^{\infty} I_{i l}^{r q}(t-s) f_{i l}^{r q}(s) e^{-\delta_{l}^{q} s} \mathrm{~d} s-\rho_{i l}^{r q} S_{i l}^{r q}\right] \mathrm{d} t \\
& +e^{\delta_{l}^{q} t}\left[\gamma_{i l}^{r q} I_{i i}^{r r}+\varrho_{l}^{q} I_{i l}^{r q}-\left(\rho_{i l}^{r q}+d_{l}^{q}\right) I_{i l}^{r q}\right] \mathrm{d} t
\end{aligned}
$$

From (3.12)-(3.14), (3.4), integrating (3.4) over $\left[t_{0}, \tau\right]$ leads to the following

$$
\begin{aligned}
V_{1} & \left(X_{00}^{00}(\tau)\right) \\
= & V_{1}\left(X_{00}^{00}\left(t_{0}\right)\right)+\sum_{r=1}^{M} \sum_{i=1}^{n_{r}} \int_{t_{0}}^{\tau} e^{\delta_{i}^{r} s}\left[B_{i}^{r}+\sum_{k \neq i}^{n_{r}} \rho_{i k}^{r r} S_{i k}^{r r}+\sum_{q \neq r}^{M} \sum_{l=1}^{n_{q}} \rho_{i a}^{r q} S_{i a}^{r q}+\varrho_{i}^{r} \int_{0}^{\infty} I_{i i}^{r r}(t-s) f_{i i}^{r r}(s) e^{-\delta_{i}^{r} s} \mathrm{~d} s\right. \\
& \left.-\left(\gamma_{i}^{r}+\sigma_{i}^{r}\right) S_{i i}^{r r}\right] \mathrm{d} s+\sum_{r=1}^{M} \sum_{i=1}^{n_{r}} \int_{t_{0}}^{\tau} e^{\delta_{i}^{r} s}\left[\sum_{k \neq i}^{n_{r}} \rho_{i k}^{r r} I_{i k}^{r r}+\sum_{q \neq r}^{M} \sum_{l=1}^{n_{q}} \rho_{i a}^{r q} I_{i a}^{r q}-\varrho_{i}^{r} I_{i i}^{r r}-\left(\gamma_{i}^{r}+\sigma_{i}^{r}+d_{i}^{r}\right) I_{i i}^{r r}\right] \mathrm{d} s \\
& +\sum_{r=1}^{M} \sum_{i=1}^{n_{r}} \sum_{j \neq i}^{n_{r}} \int_{t_{0}}^{\tau} e^{\delta_{i}^{r} s}\left[\sigma_{i j}^{r r} S_{i i}^{r r}+\varrho_{j}^{r} \int_{0}^{\infty} I_{i j}^{r r}(t-s) f_{i j}^{r r}(s) e^{-\delta_{j}^{r} s} \mathrm{~d} s-\rho_{i j}^{r r} S_{i j}^{r r}\right] \mathrm{d} s \\
& +\sum_{r=1}^{M} \sum_{i=1}^{n_{r}} \sum_{j \neq i}^{n_{r}} \int_{t_{0}}^{\tau} e^{\delta_{j}^{r} s}\left[\sigma_{i j}^{r r} I_{i i}^{r r}-\varrho_{j}^{r} I_{i j}^{r r}-\left(\rho_{i j}^{r r}+d_{j}^{r}\right) I_{i j}^{r r}\right] \mathrm{d} s \\
& +\sum_{r=1}^{M} \sum_{i=1}^{n_{r}} \sum_{q \neq r}^{n_{r}} \sum_{l=1}^{n_{q}} \int_{t_{0}}^{\tau} e^{\delta_{l}^{q} s}\left[\gamma_{i l}^{r q} S_{i i}^{r r}+\varrho_{l}^{q} \int_{0}^{\infty} I_{i l}^{r q}(t-s) f_{i l}^{r q}(s) e^{-\delta_{l}^{q} s} \mathrm{~d} s-\rho_{i l}^{r q} S_{i l}^{r q}\right] \mathrm{d} s \\
& +\sum_{r=1}^{M} \sum_{i=1}^{n_{r}} \sum_{q \neq r}^{n_{r}} \sum_{l=1}^{n_{q}} \int_{t_{0}}^{\tau} e^{\delta_{l}^{q} s}\left[\gamma_{i l}^{r q} I_{i i}^{r r}-\varrho_{l}^{q} I_{i l}^{r q}-\left(\rho_{i l}^{r q}+d_{l}^{q}\right) I_{i l}^{r q}\right] \mathrm{d} s
\end{aligned}
$$


From (3.15), we let $\tau=\tau_{k}(t)$, where $\tau_{k}(t)$ is defined in (3.9). It is easy to see from (3.15), (3.9), (3.10), and (3.11) that

$$
k=\left\|X_{00}^{00}\left(\tau_{k}(t)\right)\right\|_{1} \leq V_{1}\left(X_{00}^{00}\left(\tau_{k}(t)\right)\right)
$$

Taking the limit on (3.16) as $k \rightarrow \infty$ leads to a contradiction because the left-hand-side of the inequality (3.16) is infinite, and the right-hand-side is finite. Hence $\tau_{e}=\tau_{\infty}$ a.s. In the following, we show that $\tau_{e}=\tau_{\infty}=\infty$ a.s. We let $w \in\left\{\tau_{e}<\infty\right\}$. Applying some algebraic manipulations and simplifications to (3.15), we have the following

$$
\begin{aligned}
& I_{\left\{\tau_{e}<\infty\right\}} V_{1}\left(X_{00}^{00}(\tau)\right) \\
& =I_{\left\{\tau_{e}<\infty\right\}} V_{1}\left(X_{00}^{00}\left(t_{0}\right)\right)+I_{\left\{\tau_{e}<\infty\right\}} \sum_{r=1}^{M} \sum_{i=1}^{n_{r}} \frac{B_{i}^{r}}{\delta_{i}^{r}}\left(e^{\delta_{i}^{r} \tau}-1\right) \\
& \quad+I_{\left\{\tau_{e}<\infty\right\}} \sum_{r=1}^{M} \sum_{i=1}^{n_{r}} \sum_{q=1}^{M} \sum_{l=1}^{n_{q}} \int_{0}^{\infty} f_{i l}^{r q}(t)\left[\varrho_{l}^{q} \int_{-t}^{t_{0}} I_{i l}^{r q}(s) e^{\delta_{l}^{q_{l}} s} \mathrm{~d} s-\varrho_{l}^{q} \int_{\tau-t}^{\tau} I_{i l}^{r q}(s) e^{\delta_{l}^{q_{l}} s} \mathrm{~d} s\right] \mathrm{d} t \\
& \quad-I_{\left\{\tau_{e}<\infty\right\}} \sum_{r=1}^{M} \sum_{i=1}^{n_{r}} \int_{t_{0}}^{\tau}\left[\sigma_{i}^{r} e^{\delta_{i}^{r} s}-\sum_{j \neq i}^{n_{r}} \sigma_{i j}^{r r} e^{\delta_{j}^{r} s}\right]\left(S_{i i}^{r r}+I_{i i}^{r r}\right) \mathrm{d} s \\
& -I_{\left\{\tau_{e}<\infty\right\}} \sum_{r=1}^{M} \sum_{i=1}^{n_{r}} \int_{t_{0}}^{\tau}\left[\gamma_{i}^{r} e^{\delta_{i}^{r} s}-\sum_{q=1}^{M} \sum_{l=1}^{n_{q}} \gamma_{i l}^{r q} e^{\delta_{l}^{q_{l}} s}\right]\left(S_{i i}^{r r}+I_{i i}^{r r}\right) \mathrm{d} s \\
& \quad-I_{\left\{\tau_{e}<\infty\right\}} \sum_{r=1}^{M} \sum_{i=1}^{n_{r}} d_{i}^{r} \int_{t_{0}}^{\tau} I_{i i}^{r r} e^{\delta_{i}^{r} s} \mathrm{~d} s-I_{\left\{\tau_{e}<\infty\right\}} \sum_{r=1}^{M} \sum_{i=1}^{n_{r}} \sum_{j \neq i}^{n_{r}} d_{j}^{r} \int_{t_{0}}^{\tau} I_{i j}^{r r} e^{\delta_{j}^{r} s} \mathrm{~d} s \\
& -I_{\left\{\tau_{e}<\infty\right\}} \sum_{r=1}^{M} \sum_{i=1}^{n_{r}} \sum_{q=1}^{M} \sum_{l=1}^{n_{q}} d_{l}^{q} \int_{t_{0}}^{\tau} I_{i l}^{r q} e^{\delta_{l}^{q_{l}} s} \mathrm{~d} s,
\end{aligned}
$$

where $I_{A}$ is the indicator function of the set $A$.

We recall [30], $\sigma_{i}^{r}=\sum_{j \neq i}^{n_{r}} \sigma_{i j}^{r r}$ and $\gamma_{i}^{r}=\sum_{q \neq r}^{M} \sum_{l=1}^{n_{q}} \gamma_{i l}^{r q}$. Hence the fourth and fifth terms on the right-hand-side of (3.17) are such that

$$
\left[\sigma_{i}^{r} e^{\delta_{i}^{r} s}-\sum_{j \neq i}^{n_{r}} \sigma_{i j}^{r r} e^{\delta_{j}^{r} s}\right] \geq 0, \quad \forall \delta_{i}^{r} \geq \delta_{j}^{r}, j \neq i
$$

and

$$
\left[\gamma_{i}^{r} e^{\delta_{i}^{r} s}-\sum_{q=1}^{M} \sum_{l=1}^{n_{q}} \gamma_{i l}^{r q} e^{\delta_{l}^{q_{s}}}\right] \geq 0, \quad \forall \delta_{i}^{r} \geq \delta_{l}^{q}, q \neq r, l \in I\left(1, n_{q}\right)
$$

We now let $\tau=\tau_{k}(t) \wedge T$ in (3.17), $\exists T>0$, where $\tau_{k}(t)$ is defined in (3.9). The expected value of (3.17) is estimated as follows

$$
\begin{aligned}
& E\left[I_{\left\{\tau_{e}<\infty\right\}} V_{1}\left(X_{00}^{00}\left(\tau_{k}(t) \wedge T\right)\right)\right] \\
& \leq V_{1}\left(X_{00}^{00}\left(t_{0}\right)\right)+\sum_{i=1}^{n_{r}} \frac{B_{i}^{r}}{\delta_{i}^{r}} e^{\delta_{i}^{r} \tau_{k}(t) \wedge T} \\
& \quad+\sum_{r=1}^{M} \sum_{i=1}^{n_{r}} \sum_{q=1}^{M} \sum_{l=1}^{n_{q}} \int_{0}^{\infty} f_{i l}^{r q}(t)\left[\varrho_{l}^{q} \int_{-t}^{t_{0}} \varphi_{i l 2}^{r q}(s) e^{\delta_{l}^{q_{s}} \mathrm{~d}} \mathrm{~d}\right] \mathrm{d} t
\end{aligned}
$$

Furthermore, from (3.10), (3.11) and the definition of the indicator function $I_{A}$ it follows that

$$
I_{\left\{\tau_{e}<\infty, \tau_{k}(t) \leq T\right\}}\left\|X_{00}^{00}\left(\tau_{k}(t)\right)\right\|_{1} \leq I_{\left\{\tau_{e}<\infty\right\}} V_{1}\left(X_{00}^{00}\left(\tau_{k}(t) \wedge T\right)\right)
$$


It follows from (3.18), (3.19) and (3.9) that

$$
\begin{aligned}
& P\left(\left\{\tau_{e}<\infty, \tau_{k}(t) \leq T\right\}\right) k \\
& =E\left[I_{\left\{\tau_{e}<\infty, \tau_{k}(t) \leq T\right\}}\left\|X_{00}^{00}\left(\tau_{k}(t)\right)\right\|_{1}\right] \leq E\left[I_{\left\{\tau_{e}<\infty\right\}} V\left(X_{00}^{00}\left(\tau_{k}(t) \wedge T\right)\right)\right] \\
& \leq V_{1}\left(X_{00}^{00}\left(t_{0}\right)\right)+\sum_{i=1}^{n_{r}} \frac{B_{i}^{r}}{\delta_{i}^{r}} e^{\delta_{i}^{r} T}+\sum_{r=1}^{M} \sum_{i=1}^{n_{r}} \sum_{q=1}^{M} \sum_{l=1}^{n_{q}} \int_{0}^{\infty} f_{i l}^{r q}(t)\left[\varrho_{l}^{q} \int_{-t}^{t_{0}} \varphi_{i l 2}^{r q}(s) e^{\delta_{l}^{q} s} \mathrm{~d} s\right] \mathrm{d} t
\end{aligned}
$$

It follows immediately from (3.20) that $P\left(\left\{\tau_{e}<\infty, \tau_{\infty} \leq T\right\}\right) \rightarrow 0$ as $k \rightarrow \infty$. Furthermore, since $T<\infty$ is arbitrary, we conclude that $P\left(\left\{\tau_{e}<\infty, \tau_{\infty}<\infty\right\}\right)=0$. Finally, by the total probability principle,

$$
\begin{aligned}
P\left(\left\{\tau_{e}<\infty\right\}\right) & =P\left(\left\{\tau_{e}<\infty, \tau_{\infty}=\infty\right\}\right)+P\left(\left\{\tau_{e}<\infty, \tau_{\infty}<\infty\right\}\right) \\
& \leq P\left(\left\{\tau_{e} \neq \tau_{\infty}\right\}\right)+P\left(\left\{\tau_{e}<\infty, \tau_{\infty}<\infty\right\}\right)=0 .
\end{aligned}
$$

Thus from (3.21), $\tau_{e}=\tau_{\infty}=\infty$ a.s. as was required to show.

Remark 3.1. For any $r \in I(1, M)$ and $i \in I\left(1, n_{r}\right)$, Theorem 3.1 signifies that the number of residents of site $s_{i}^{r}$ of all categories present at home site $s_{i}^{r}$, or visiting intra and inter-regional sites $s_{j}^{r}$ and $s_{l}^{q}$ respectively, are nonnegative. This implies that the total number of residents of site $s_{i}^{r}$ present at home site and also visiting sites in regions in their intra and inter-regional accessible domains [21], given by the sum $N_{i 0}^{r r}(t)=\sum_{u=1}^{M} \sum_{a=1}^{n_{u}} y_{i a}^{r u}$, is nonnegative. Moreover, the total effective population [21], defined by eff $\left(N_{i 0}^{r r}\right)(t)=\sum_{u=1}^{M} \sum_{a=1}^{n_{u}} y_{a i}^{u r}$, at any site $s_{i}^{r}$ in region $C_{r}$ is also nonnegative at all time $t \geq t_{0}$.

The following result defines an upper bound for the solution process of the system (2.1)-(2.3). We utilize Theorem 3.1 to establish this result.

Theorem 3.2. Suppose the hypotheses of Theorem 3.1 is satisfied. Let $\mu=\min _{1 \leq u \leq M, 1 \leq a \leq n_{u}}\left(\delta_{a}^{u}\right)$. If

$$
\sum_{r=1}^{M} \sum_{u=1}^{M} \sum_{i=1}^{n_{r}} \sum_{a=1}^{n_{u}} y_{i a}^{r u}\left(t_{0}\right) \leq \frac{1}{\mu} \sum_{r=1}^{M} \sum_{i=1}^{n_{r}} B_{i}^{r},
$$

then

$$
\sum_{r=1}^{M} \sum_{u=1}^{M} \sum_{i=1}^{n_{r}} \sum_{a=1}^{n_{u}} y_{i a}^{r u}(t) \leq \frac{1}{\mu} \sum_{r=1}^{M} \sum_{i=1}^{n_{r}} B_{i}^{r}, \text { for } t \geq t_{0} \text { a.s. }
$$

Proof: See ([22], Lemma 3.2)

Remark 3.2. From Theorem 3.1 and Theorem 3.2, we conclude that a closed ball $\overline{\mathfrak{B}}_{R^{3 n^{2}}}(\overrightarrow{0} ; r)$ in $R^{3 n^{2}}$ under the sum norm $\|\cdot\|_{1}$ centered at the origin $\overrightarrow{0} \in R^{3 n^{2}}$, with radius $r=\frac{1}{\mu} \sum_{r=1}^{M} \sum_{i=1}^{n_{r}} B_{i}^{r}$ is self-invariant with regard to a twoscale network dynamics of human epidemic process (2.1)-(2.3) that is under the influence of human mobility process [30]. That is,

$$
\overline{\mathfrak{B}}_{R^{3 n^{2}}}(\overrightarrow{0} ; r)=\left\{\left(S_{i a}^{r u}, I_{i a}^{r u}, R_{i a}^{r u}\right): y_{i a}^{r u}(t) \geq 0 \text { and }\left\|x_{00}^{00}\right\|_{1}=\sum_{r=1}^{M} \sum_{u=1}^{M} \sum_{i=1}^{n_{r}} \sum_{a=1}^{n_{u}} y_{i a}^{r u}(t) \leq \frac{1}{\mu} \sum_{r=1}^{M} \sum_{i=1}^{n_{r}} B_{i}^{r}\right\}
$$

is a positive self-invariant set for system (2.1)-(2.3). We shall denote

$$
\bar{B} \equiv \frac{1}{\mu} \sum_{r=1}^{M} \sum_{i=1}^{n_{r}} B_{i}^{r}
$$




\section{Existence and Asymptotic Behavior of Disease Free Equilibrium}

In this section, we study the existence and the asymptotic behavior of the disease free equilibrium state of the system (2.1)-(2.3). The disease free equilibrium is obtained by solving the system of algebraic equations obtained by setting the drift and the diffusion parts of the system of stochastic differential equations to zero. In addition, we utilize the conditions that $I=R=0$ in the event when there is no disease in the population. We summarize the results in the following. For any $r, u \in I(1, M), i \in I\left(1, n_{r}\right)$ and $a \in I\left(1, n_{u}\right)$, let

$$
D_{i}^{r}=\gamma_{i}^{r}+\sigma_{i}^{r}+\delta_{i}^{r}-\sum_{a=1}^{n_{r}} \frac{\rho_{i a}^{r r} \sigma_{i a}^{r r}}{\rho_{i a}^{r r}+\delta_{a}^{r}}-\sum_{u \neq r}^{M} \sum_{a=1}^{n_{u}} \frac{\rho_{i a}^{r r} \gamma_{i a}^{r u}}{\rho_{i a}^{r u}+\delta_{a}^{u}} \geq \delta_{i}^{r}>0 .
$$

Furthermore, let $\left(S_{i a}^{r u^{*}}, I_{i a}^{r u *}, R_{i a}^{r u *}\right)$ be the equilibrium state of the delayed system (2.1)-(2.3). One can see that the disease free equilibrium state is given by $E_{i a}^{r u}=\left(S_{i a}^{r u *}, 0,0\right)$, where

$$
S_{i a}^{r u *}= \begin{cases}\frac{B_{i}^{r}}{D_{i}^{r}}, & \text { for } u=r, a=i, \\ \frac{B_{i}^{r}}{D_{i}^{r}} \frac{\sigma_{i j}^{r r}}{\rho_{i j}^{r r}+\delta_{j}^{r}}, & \text { for } u=r, a \neq i, \\ \frac{B_{i}^{r}}{D_{i}^{r}} \frac{\gamma_{i a}^{r u}}{\rho_{i a}^{r u}+\delta_{a}^{u}}, & \text { for } u \neq r .\end{cases}
$$

The asymptotic stability property of $E_{i a}^{r u}$ will be established by verifying the conditions of the stochastic version of the Lyapunov second method given in ([31], Theorem 2.4), [32], and ([31], Theorem 4.4), [32] respectively. In order to study the qualitative properties of (2.1)-(2.3) with respect to the equilibrium state $\left(S_{i a}^{r u *}, 0,0\right)$, first, we use the change of variable that shifts the equilibrium to the origin. For this purpose, we use the following transformation:

$$
\left\{\begin{array}{l}
U_{i a}^{r u}=S_{i a}^{r u}-S_{i a}^{r u^{*}} \\
V_{i a}^{r u}=I_{i a}^{r u} \\
W_{i a}^{r u}=R_{i a}^{r u}
\end{array}\right.
$$

By employing this transformation, system (2.1)-(2.3) is transformed into the following forms

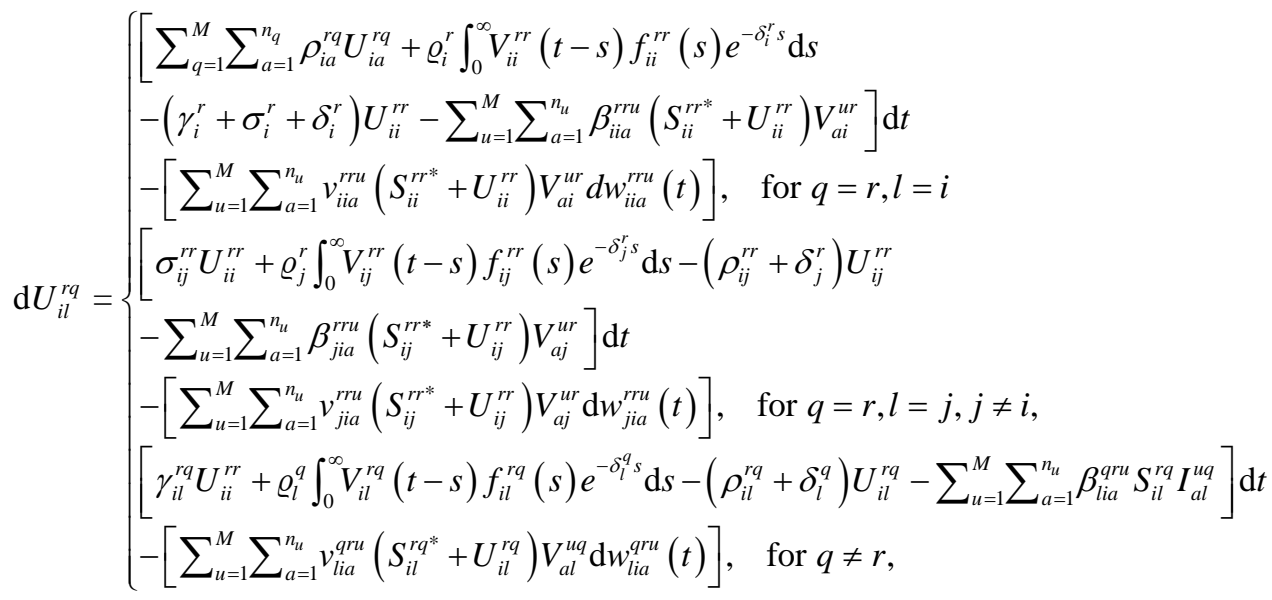




$$
\mathrm{d} V_{i l}^{r q}=\left\{\begin{array}{l}
{\left[\begin{array}{l}
{\left[\sum_{q=1}^{M} \sum_{a=1}^{n_{q}} \rho_{i a}^{r q} V_{i a}^{r q}-\left(\varrho_{i}^{r}+\gamma_{i}^{r}+\sigma_{i}^{r}+\delta_{i}^{r}+d_{i}^{r}\right) W_{i i}^{r r}\right.} \\
\left.+\sum_{u=1}^{M} \sum_{a=1}^{n_{u}} \beta_{i i a}^{r r u}\left(S_{i i}^{r r *}+U_{i i}^{r r}\right) V_{a i}^{u r}\right] \mathrm{d} t \\
+\left[\sum_{u=1}^{M} \sum_{a=1}^{n_{u}} V_{i i a}^{r r u}\left(S_{i i}^{r r *}+U_{i i}^{r r}\right) V_{a i}^{u r} \mathrm{~d} w_{i i a}^{r r u}(t)\right], \text { for } q=r, l=i \\
{\left[\sigma_{i j}^{r r} V_{i i}^{r r}-\left(\varrho_{j}^{r}+\rho_{i j}^{r r}+\delta_{j}^{r}+d_{j}^{r}\right) V_{i j}^{r r}+\sum_{u=1}^{M} \sum_{a=1}^{n_{u}} \beta_{j i a}^{r r u}\left(S_{i j}^{r r *}+U_{i j}^{r r}\right) V_{a j}^{u r}\right] \mathrm{d} t} \\
+\left[\sum_{u=1}^{M} \sum_{a=1}^{n_{u}} v_{j i a}^{r r u}\left(S_{i j}^{r r^{*}}+U_{i j}^{r r}\right) V_{a j}^{u r} \mathrm{~d} w_{j i a}^{r r u}(t)\right], \text { for } q=r, l=j, j \neq i, \\
{\left[\gamma_{i l}^{r q} V_{i i}^{r r}-\left(\varrho_{l}^{q}+\rho_{i l}^{r q}+\delta_{l}^{q}+d_{l}^{q}\right) V_{i l}^{r q} \sum_{u=1}^{M} \sum_{a=1}^{n_{u}} \beta_{l i a}^{q r u}\left(S_{i l}^{r q^{*}}+U_{i l}^{r q}\right) V_{a l}^{u q}\right] \mathrm{d} t} \\
+\left[\sum_{u=1}^{M} \sum_{a=1}^{n_{u}} v_{l i a}^{q r u}\left(S_{i l}^{r q^{*}}+U_{i l}^{r q}\right) V_{a l}^{u q} \mathrm{~d} w_{l i a}^{q r u}(t)\right], \text { for } q \neq r,
\end{array}\right.}
\end{array}\right.
$$

and

$$
\mathrm{d} W_{i l}^{r q}=\left\{\begin{array}{l}
{\left[\sum_{q=1}^{M} \sum_{a=1}^{n_{q}} \rho_{i l}^{r q} W_{i l}^{r q}+\varrho_{i}^{r} V_{i i}^{r r}-\varrho_{i}^{r} \int_{0}^{\infty} V_{i i}^{r r}(t-s) f_{i i}^{r r}(s) e^{-\delta_{i}^{r} s} \mathrm{~d} s-\left(\gamma_{i}^{r}+\sigma_{i}^{r}+\delta_{i}^{r}\right) W_{i i}^{r r}\right] \mathrm{d} t, \text { for } q=r, l=i} \\
{\left[\sigma_{i j}^{r r} W_{i i}^{r r}+\varrho_{j}^{r} V_{i j}^{r r}-\varrho_{j}^{r} \int_{0}^{\infty} V_{i j}^{r r}(t-s) f_{i j}^{r r}(s) e^{-\delta_{j}^{r} s} \mathrm{~d} s-\left(\rho_{i j}^{r r}+\delta_{j}^{r}\right) W_{i j}^{r r}\right] \mathrm{d} t, \text { for } q=r, l=j, j \neq i} \\
{\left[\gamma_{i l}^{r q} W_{i i}^{r r}+\varrho_{l}^{q} V_{i l}^{r q}-\varrho_{l}^{q} \int_{0}^{\infty} V_{i l}^{r q}(t-s) f_{i l}^{r q}(s) e^{-\delta_{l}^{q} s} \mathrm{~d} s-\left(\rho_{i l}^{r q}+\delta_{l}^{q}\right) W_{i l}^{r q}\right] \mathrm{d} t, \text { for } q \neq r}
\end{array}\right.
$$

We state and prove the following lemmas that would be useful in the proofs of the stability results.

Lemma 4.1. Let $V_{1}: \mathbb{R}^{3 n^{2}} \times \mathbb{R}_{+} \rightarrow \mathbb{R}_{+}$be a function defined by

$$
\left\{\begin{array}{l}
V_{1}\left(\tilde{x}_{00}^{00}\right)=\sum_{r=1}^{M} \sum_{u=1}^{M} \sum_{i=1}^{n_{r}} \sum_{a=1}^{n_{u}} V\left(\tilde{x}_{i a}^{r u}\right), \\
V_{1}\left(\tilde{x}_{i a}^{r u}\right)=\left(S_{i a}^{r u}-S_{i a}^{r u^{*}}+I_{i a}^{r u}\right)^{2}+c_{i a}^{r u}\left(I_{i a}^{r u}\right)^{2}+\left(R_{i a}^{r u}\right)^{2} \\
\tilde{x}_{00}^{00}=\left(U_{i a}^{r u}, V_{i a}^{r u}, W_{i a}^{r u}\right)^{\mathrm{T}} \text { and } c_{i a}^{r u} \geq 0 .
\end{array}\right.
$$

Then $V_{1} \in \mathcal{C}^{2,1}\left(\mathbb{R}^{3 n^{2}} \times \mathbb{R}_{+}, \mathbb{R}_{+}\right)$, and it satisfies

$$
b\left(\left\|\tilde{x}_{00}^{00}\right\|\right) \leq V_{1}\left(\tilde{x}_{00}^{00}(t)\right) \leq a\left(\left\|\tilde{x}_{00}^{00}\right\|\right)
$$

where

$$
\begin{aligned}
& b\left(\left\|\tilde{x}_{00}^{00}\right\|\right)=\min _{1 \leq r, u \leq M, 1 \leq i \leq n_{r}, 1 \leq a \leq n_{u}}\left\{\frac{c_{i a}^{r u}}{2+c_{i a}^{r u}}\right\} \sum_{r=1}^{M} \sum_{u=1}^{M} \sum_{i=1}^{n_{r}} \sum_{a=1}^{n_{u}}\left[\left(U_{i a}^{r u}\right)^{2}+\left(V_{i a}^{r u}\right)^{2}+\left(W_{i a}^{r u}\right)^{2}\right] \\
& a\left(\left\|\tilde{x}_{00}^{00}\right\|\right)=\max _{1 \leq r, u \leq M, 1 \leq i \leq n_{r}, 1 \leq a \leq n_{u}}\left\{c_{i a}^{r u}+2\right\} \sum_{r=1}^{M} \sum_{u=1}^{M} \sum_{i=1}^{n_{r}} \sum_{a=1}^{n_{u}}\left[\left(U_{i a}^{r u}\right)^{2}+\left(V_{i a}^{r u}\right)^{2}+\left(W_{i a}^{r u}\right)^{2}\right] .
\end{aligned}
$$

Proof: See ([22], Lemma 4.1).

Remark 4.1. Lemma 4.1 shows that the Lyapunov function $V$ defined in (4.7) is positive definite, decrescent and radially unbounded (4.8) function [31] [32].

We now state the following lemma.

Lemma 4.2. Assume that the hypothesis of Lemma 4.1 is satisfied. Define a Lyapunov functional

$$
V=V_{1}+V_{2},
$$

where $V_{1}$ is defined by (4.7), and 


$$
V_{2}=3 \sum_{r=1}^{M} \sum_{i=1}^{n_{r}} \sum_{u=1}^{M} \sum_{a=1}^{n_{u}}\left[\frac{\left(\varrho_{a}^{u}\right)^{2}}{\mu_{i a}^{r u}} \int_{0}^{\infty}\left(f_{i a}^{r r}(s) e^{-2 \delta_{a}^{r} s} \int_{t-s}^{t}\left(V_{i a}^{r u}(\theta)\right)^{2} \mathrm{~d} \theta\right) \mathrm{d} s\right],
$$

Furthermore, let

$$
\mathfrak{U}_{i a}^{r u}=\left\{\begin{array}{l}
\frac{\left[\frac{\left.\sum_{u=1}^{M} \sum_{a=1}^{n_{u}} \mu_{i a}^{r u}+\sum_{a \neq i}^{n_{r}} \frac{\left(\sigma_{i a}^{r r}\right)^{2}}{\mu_{i i}^{r r}}+\sum_{a \neq r}^{M} \sum_{a=1}^{n_{r}} \frac{\left(\gamma_{i a}^{r u}\right)^{2}}{\mu_{i i}^{r r}}+2 \mu_{i i}^{r r}\right]}{\left.\mu_{i a}^{r r}+\gamma_{i i}^{r}+\sigma_{i}^{r}+\delta_{i}^{r}\right)}, \mu_{i a}^{r r}\right]}{\left(\rho_{i a}^{r r}+\delta_{a}^{r}\right)}, \text { for } u=r, a \neq i \\
\frac{\left[\frac{\left(\rho_{i a}^{r u}\right)^{2}}{\mu_{i a}^{r u}}+\mu_{i i}^{r r}+\frac{3}{2} \mu_{i a}^{r u}\right]}{\left(\rho_{i a}^{r u}+\delta_{a}^{u}\right)}, \text { for } u \neq r,
\end{array}\right.
$$

$$
\mathfrak{V}_{i a}^{r u}=\left\{\begin{array}{l}
\frac{\frac{1}{2} \sum_{u=1}^{M} \sum_{a=1}^{n_{u}} \mu_{i a}^{r u}+\frac{1}{2} \sum_{v=1}^{M} \sum_{b=1}^{n_{v}} \beta_{i i b}^{r r v}\left(S_{i i}^{r r *} \mu_{i i}^{r r}+\mu_{i i}^{r r}\right)+\frac{1}{2} d_{i i}^{r r}}{\varrho_{i}^{r}+\gamma_{i}^{r}+\sigma_{i}^{r}+\delta_{i}^{r}+d_{i}^{r}}, \text { for } a=i, u=r \\
\frac{\frac{1}{2} \mu_{i i}^{r r}+\frac{1}{2} \sum_{v=1}^{M} \sum_{b=1}^{n_{v}} \beta_{a i b}^{r v}\left(S_{i a}^{r *} \mu_{i a}^{r r}+\mu_{i a}^{r r}\right)+\frac{1}{2} d_{a i}^{r r}}{\varrho_{a}^{r}+\rho_{i a}^{r r}+\delta_{a}^{r}+d_{a}^{r}}, \text { for } a \neq i, u=r \\
\frac{\frac{1}{2} \mu_{i i}^{r r}+\frac{1}{2} \sum_{v=1}^{M} \sum_{b=1}^{n_{v}} \beta_{a i b}^{u r v}\left(S_{i i}^{r u *} \mu_{i a}^{r u}+\mu_{i a}^{r u}\right)+\frac{1}{2} d_{a i}^{u r}}{\varrho_{a}^{u}+\rho_{i a}^{r u}+\delta_{a}^{u}+d_{a}^{u}}, \text { for } u \neq r .
\end{array}\right.
$$

and

$$
\mathfrak{W}_{i a}^{r u}=\left\{\begin{array}{l}
\frac{\left[\frac{1}{2} \sum_{u=1}^{M} \sum_{a=1}^{n_{u}} \mu_{i a}^{r u}+\frac{1}{2} \sum_{u \neq r}^{M} \sum_{a=1}^{n_{r}} \frac{\left(\gamma_{i a}^{r u}\right)^{2}}{\mu_{i i}^{r r}}+\frac{1}{2} \sum_{a \neq i}^{n_{r}} \frac{\left(\sigma_{i a}^{r r}\right)^{2}}{\mu_{i i}^{r r}}+\mu_{i i}^{r r}\right]}{\left(\gamma_{i}^{r}+\sigma_{i}^{r}+\delta_{i}^{r}\right)}, \text { for } u=r, a=i, \\
\frac{\left[\frac{1}{2} \frac{\left(\rho_{i a}^{r r}\right)^{2}}{\mu_{i a}^{r r}}+\frac{1}{2} \mu_{i i}^{r r}+\mu_{i a}^{r r}\right]}{\left(\rho_{i a}^{r r}+\delta_{a}^{r}\right)}, \text { for } u=r, a \neq i, \\
\frac{\left[\frac{1}{2} \frac{\left(\rho_{i a}^{r u}\right)^{2}}{\mu_{i a}^{r u}}+\frac{1}{2} \mu_{i i}^{r r}+\mu_{i a}^{r u}\right]}{\left(\rho_{i a}^{r u}+\delta_{a}^{u}\right)}, \text { for } u \neq r
\end{array}\right.
$$

for some suitably defined positive numbers $\mu_{i a}^{r u}$ and $d_{a i}^{u r}$, where $\mu_{i a}^{r u}$ depends on $\delta_{a}^{u}$, for all $r, u \in I^{r}(1, M), i \in I(1, n)$ and $a \in I_{i}^{r}\left(1, n_{r}\right)$. Assume that $\mathfrak{U}_{i a}^{r u} \leq 1, \mathfrak{V}_{i a}^{r u}<1$ and $\mathfrak{W}_{i a}^{r u} \leq 1$. There exist positive numbers $\phi_{i a}^{r u}, \psi_{i a}^{r u}$ and $\varphi_{i a}^{r u}$ such that the differential operator $L V$ associated with Ito-Doob type stochastic system (2.1)-(2.3) satisfies the following inequality 


$$
\begin{aligned}
L V\left(\tilde{X}_{00}^{00}\right) \leq & \sum_{r=1}^{M} \sum_{i=1}^{n_{r}}\left[-\left[\phi_{i i}^{r r}\left(U_{i i}^{r r}\right)^{2}+\psi_{i i}^{r r}\left(V_{i i}^{r r}\right)^{2}+\varphi_{i i}^{r r}\left(W_{i i}^{r r}\right)^{2}\right]\right. \\
& -\sum_{a \neq i}^{n_{r}}\left[\phi_{i a}^{r r}\left(U_{i a}^{r r}\right)^{2}+\psi_{i a}^{r r}\left(V_{i a}^{r r}\right)^{2}+\varphi_{i a}^{r r}\left(W_{i a}^{r r}\right)^{2}\right] \\
& \left.-\sum_{u \neq r}^{M} \sum_{a=1}^{n_{u}}\left[\phi_{i a}^{r u}\left(U_{i a}^{r r}\right)^{2}+\psi_{i a}^{r u}\left(V_{i a}^{r u}\right)^{2}+\varphi_{i a}^{r u}\left(W_{i a}^{r u}\right)^{2}\right]\right] .
\end{aligned}
$$

Moreover,

$$
L V\left(\tilde{x}_{00}^{00}\right) \leq-c V_{1}\left(\tilde{x}_{00}^{00}\right)
$$

where a positive constant $c$ is defined by

$$
C=\frac{\min _{1 \leq r, u \leq M, 1 \leq i \leq n_{r}, 1 \leq a \leq n_{u}}\left\{\phi_{i a}^{r u}, \psi_{i a}^{r u}, \varphi_{i a}^{r u}\right\}}{\max _{1 \leq r, u \leq M, 1 \leq i \leq n_{r}, 1 \leq a \leq n_{u}}\left\{C_{i a}^{r u}+2\right\}}
$$

Proof:

The computation of differential operator [31] [32] applied to the Lyapunov function $V_{1}$ in (4.7) with respect to the large-scale system of Ito-Doob type stochastic differential Equations (2.1)-(2.3) is as follows:

$$
L V_{1}\left(\tilde{x}_{00}^{00}\right)=\sum_{r=1}^{M} \sum_{i=1}^{n_{r}}\left[L V_{1}\left(\tilde{x}_{i i}^{r r}\right)+\sum_{j \neq i}^{n_{r}} L V_{1}\left(\tilde{x}_{i j}^{r r}\right)+\sum_{u \neq r}^{M} \sum_{a=1}^{n_{u}} L V_{1}\left(\tilde{x}_{i a}^{r u}\right)\right],
$$

where,

$$
\begin{aligned}
& L V_{1}\left(\tilde{X}_{i i}^{r r}\right)=2 \sum_{u=1}^{M} \sum_{a=1}^{n_{u}}\left[\left(1+C_{i i}^{r r}\right) \rho_{i a}^{r u} V_{i a}^{r u} V_{i i}^{r r}+\rho_{i a}^{r u} U_{i a}^{r u} U_{i i}^{r r}+\rho_{i a}^{r u} V_{i a}^{r u} U_{i i}^{r r}+\rho_{i a}^{r u} U_{i a}^{r u} V_{i i}^{r r}+\rho_{i a}^{r u} W_{i a}^{r u} W_{i i}^{r r}\right] \\
& +2 \varrho_{i}^{r} U_{i i}^{r r} \int_{0}^{\infty} V_{i i}^{r r}(t-s) f_{i i}^{r r}(s) e^{-\delta_{i}^{r} s} \mathrm{~d} s+2 \varrho_{i}^{r} V_{i i}^{r r} \int_{0}^{\infty} V_{i i}^{r r}(t-s) f_{i i}^{r r}(s) e^{-\delta_{i}^{r} s} \mathrm{~d} s \\
& -2 \varrho_{i}^{r} W_{i i}^{r r} \int_{0}^{\infty} V_{i i}^{r r}(t-s) f_{i i}^{r r}(s) e^{-\delta_{i}^{r} s} \mathrm{~d} s-2 \varrho_{i}^{r} V_{i i}^{r r} W_{i i}^{r r} \\
& -2\left[\left(\varrho_{i}^{r}+d_{i}^{r}\right)+2\left(\gamma_{i}^{r}+\sigma_{i}^{r}+\delta_{i}^{r}\right)\right] V_{i i}^{r r} U_{i i}^{r r}-2\left(\gamma_{i}^{r}+\sigma_{i}^{r}+\delta_{i}^{r}\right)\left(U_{i i}^{r r}\right)^{2} \\
& -2\left[\left(c_{i i}^{r r}+1\right) \varrho_{i}^{r}+2\left(c_{i i}^{r r}+1\right)\left(\gamma_{i}^{r}+\sigma_{i}^{r}+\delta_{i}^{r}+d_{i}^{r}\right)\right]\left(V_{i i}^{r r}\right)^{2} \\
& -2\left(\gamma_{i}^{r}+\sigma_{i}^{r}+\alpha_{i}^{r}+\delta_{i}^{r}\right)\left(W_{i i}^{r r}\right)^{2}+2 c_{i i}^{r r} \sum_{u=1}^{M} \sum_{a=1}^{n_{u}} \beta_{i i a}^{r r u}\left(S_{i i}^{r r *}+U_{i i}^{r r}\right) V_{a i}^{u r} V_{i i}^{r r} \\
& +c_{i i}^{r r} \sum_{u=1}^{M} \sum_{a=1}^{n_{u}}\left(v_{i i a}^{r r u}\right)^{2}\left(S_{i i}^{r r *}+U_{i i}^{r r}\right)^{2}\left(V_{a i}^{u r}\right)^{2} \text {, for } u=r, a=i \\
& \sum_{a \neq i}^{n_{r}} L V_{1}\left(\tilde{X}_{i a}^{r r}\right)=\sum_{a \neq r}^{n_{r}}\left\{2\left(1+c_{i a}^{r r}\right) \sigma_{i a}^{r r} V_{i a}^{r r} V_{i i}^{r r}+2 \sigma_{i a}^{r r} U_{i a}^{r r} U_{i i}^{r r}+2 \sigma_{i a}^{r r} V_{i a}^{r r} U_{i i}^{r r}+2 \sigma_{i a}^{r r} U_{i a}^{r r} V_{i i}^{r r}+2 \sigma_{i a}^{r r} W_{i a}^{r r} W_{i i}^{r r}\right. \\
& +2 \varrho_{a}^{r} U_{i a}^{r r} \int_{0}^{\infty} V_{i a}^{r r}(t-s) f_{i a}^{r r}(s) e^{-\delta_{a}^{r} s} \mathrm{~d} s+2 \rho_{a}^{r} V_{i a}^{r r} \int_{0}^{\infty} V_{i a}^{r r}(t-s) f_{i a}^{r r}(s) e^{-\delta_{a}^{r} s} \mathrm{~d} s \\
& -2 \varrho_{a}^{r} W_{i a}^{r r} \int_{0}^{\infty} V_{i a}^{r r}(t-s) f_{i a}^{r r}(s) e^{-\delta_{a}^{r} s} \mathrm{~d} s-2\left[\left(c_{i a}^{r r}+1\right) \varrho_{a}^{r}+2\left(c_{i a}^{r r}+1\right)\left(\rho_{i a}^{r r}+\delta_{a}^{r}\right)\right]\left(V_{i a}^{r r}\right)^{2} \\
& -2\left(\rho_{i a}^{r r}+\delta_{a}^{r}\right)\left(U_{i a}^{r r}\right)^{2}-2\left(\rho_{i a}^{r r}+\delta_{a}^{r}\right)\left(W_{i a}^{r r}\right)^{2}+2 \varrho_{a}^{r} V_{i a}^{r r} W_{i a}^{r r} \\
& \left.-2\left[\left(\varrho_{a}^{r}+d_{a}^{r}\right)+2\left(\rho_{i a}^{r r}+\delta_{a}^{r}\right)\right] V_{i a}^{r r} U_{i a}^{r r}\right\}+2 \sum_{a \neq i}^{n_{r}} c_{i a}^{r r} \sum_{v=1}^{M} \sum_{b=1}^{n_{v}} \beta_{a i b}^{r r v}\left(S_{i a}^{r r *}+U_{i a}^{r r}\right) V_{b a}^{v r} V_{i a}^{r r} \\
& +\sum_{a \neq i}^{n_{r}} c_{i a}^{r r} \sum_{v=1}^{M} \sum_{b=1}^{n_{v}}\left(v_{a i b}^{r r v}\right)^{2}\left(S_{i a}^{r r *}+U_{i a}^{r r}\right)^{2}\left(V_{b a}^{v r}\right)^{2} \text {, for } u=r, a \neq i
\end{aligned}
$$




$$
\begin{aligned}
\sum_{u \neq r a=1}^{M} \sum_{r}^{n_{r}} L V_{1}\left(\tilde{X}_{i a}^{r u}\right)= & \sum_{u \neq r}^{M} \sum_{a=1}^{n_{u}}\left\{2\left(1+c_{i a}^{r u}\right) \gamma_{i a}^{r u} V_{i a}^{r u} V_{i i}^{r r}+2 \gamma_{i a}^{r u} U_{i a}^{r u} U_{i i}^{r r}+2 \gamma_{i a}^{r u} V_{i a}^{r u} U_{i i}^{r r}+2 \gamma_{i a}^{r u} U_{i a}^{r u} V_{i i}^{r r}\right. \\
& +2 \gamma_{i a}^{r u} W_{i a}^{r u} W_{i i}^{r r}+2 \varrho_{a}^{u} U_{i a}^{r u} \int_{0}^{\infty} V_{i a}^{r u}(t-s) f_{i a}^{r u}(s) e^{-\delta_{a}^{u} s} \mathrm{~d} s \\
& +2 \varrho_{a}^{u} V_{i a}^{r u} \int_{0}^{\infty} V_{i a}^{r u}(t-s) f_{i a}^{r u}(s) e^{-\delta_{a}^{u} s} \mathrm{~d} s-2 \varrho_{a}^{u} W_{i a}^{r u} \int_{0}^{\infty} V_{i a}^{r u}(t-s) f_{i a}^{r u}(s) e^{-\delta_{a}^{u} s} \mathrm{~d} s \\
& -2\left[\left(c_{i a}^{r u}+1\right) \varrho_{a}^{u}+2\left(c_{i a}^{r u}+1\right)\left(\rho_{i a}^{r u}+\delta_{a}^{u}+d_{a}^{u}\right)\right]\left(V_{i a}^{r u}\right)^{2}-2\left(\rho_{i a}^{r u}+\delta_{a}^{u}\right)\left(U_{i a}^{r u}\right)^{2} \\
& \left.-2\left(\rho_{i a}^{r u}+\alpha_{a}^{u}+\delta_{a}^{u}\right)\left(W_{i a}^{r r}\right)^{2}+2 \varrho_{a}^{u} V_{i a}^{r u} W_{i a}^{r u}-2\left[\left(\varrho_{a}^{u}+d_{a}^{u}\right)+2\left(\rho_{i a}^{r u}+\delta_{a}^{u}\right)\right] V_{i a}^{r u} U_{i a}^{r u}\right\} \\
& +2 \sum_{u \neq r a=1}^{M} \sum_{i a}^{n_{u}} c_{i a}^{r u} \sum_{v=1}^{M} \sum_{b=1}^{n_{v}} \beta_{a i b}^{u r v}\left(S_{i a}^{r u *}+U_{i a}^{r u}\right) V_{b a}^{v u} V_{i a}^{r u} \\
& +\sum_{u \neq r}^{M} \sum_{a=1}^{n_{r}} c_{i a}^{r u} \sum_{v=1}^{M} \sum_{v=1}^{n_{v}}\left(v_{a i b}^{u r v}\right)^{2}\left(S_{i a}^{r u^{*}}+U_{i a}^{r u}\right)^{2}\left(V_{b a}^{v u}\right)^{2}, \text { for } u \neq r
\end{aligned}
$$

By using (3.25) and the algebraic inequality

$$
2 a b \leq \frac{a^{2}}{g(c)}+b^{2} g(c)
$$

where $a, b, c \in \mathbb{R}$, and the function $g$ is such that $g(c)>0$. The fourteenth term in (4.19)-(4.21) is estimated as follows:

$$
\begin{aligned}
& 2 \sum_{v=1}^{M} \sum_{b=1}^{n_{v}} c_{i i}^{r r} \beta_{i i b}^{r v}\left(S_{i i}^{r *}+U_{i i}^{r r}\right) V_{b i}^{v r} V_{i i}^{r r} \\
& \leq \sum_{v=1}^{M} \sum_{b=1}^{n_{v}} c_{i i}^{r r} \beta_{i i b}^{r r v}\left(S_{i i}^{r r *} g_{i}^{r}\left(\delta_{i}^{r}\right)+g_{i}^{r}\left(\delta_{i}^{r}\right)\right)\left(V_{i i}^{r r}\right)^{2} \\
& +\sum_{v=1}^{M} \sum_{b=1}^{n_{v}} c_{i i}^{r r} \beta_{i i b}^{r r v}\left(\frac{S_{i i}^{r * *}}{g_{i}^{r}\left(\delta_{i}^{r}\right)}+\frac{\bar{B}^{2}}{g_{i}^{r}\left(\delta_{i}^{r}\right)}\right)\left(V_{b i}^{v r}\right)^{2} \\
& 2 \sum_{a \neq r}^{n_{r}} \sum_{v=1}^{M} \sum_{b=1}^{n_{v}} c_{i a}^{r r} \beta_{a i b}^{r v}\left(S_{i a}^{r r *}+U_{i a}^{r r}\right) V_{b a}^{v r} V_{i a}^{r r} \\
& \leq \sum_{a \neq r}^{n_{r}} \sum_{v=1}^{M} \sum_{b=1}^{n_{v}} c_{i a}^{r r} \beta_{a i b}^{r v}\left(S_{i a}^{r r^{*} *} g_{i}^{r}\left(\delta_{a}^{r}\right)+g_{i}^{r}\left(\delta_{a}^{r}\right)\right)\left(V_{i a}^{r r}\right)^{2} \\
& +\sum_{a \neq r}^{n_{r}} \sum_{v=1}^{M} \sum_{b=1}^{n_{v}} c_{i a}^{r r} \beta_{a i b}^{r r v}\left(\frac{S_{i a}^{r * *}}{g_{i}^{r}\left(\delta_{a}^{r}\right)}+\frac{\bar{B}^{2}}{g_{i}^{r}\left(\delta_{a}^{r}\right)}\right)\left(V_{b i}^{v r}\right)^{2}
\end{aligned}
$$

and

$$
\begin{aligned}
& 2 \sum_{u \neq r}^{M} \sum_{a=1}^{n_{u}} \sum_{v=1}^{M} \sum_{b=1}^{n_{v}} c_{i a}^{r u} \beta_{a i b}^{u r v}\left(S_{i a}^{r u^{*}}+U_{i a}^{r u}\right) V_{b a}^{v u} V_{i a}^{r u} \\
& \leq \sum_{u \neq r}^{M} \sum_{a=1}^{n_{u}} \sum_{v=1}^{M} \sum_{b=1}^{n_{v}} c_{i a}^{r u} \beta_{a i b}^{u r v}\left(S_{i a}^{r u^{*}} g_{i}^{r}\left(\delta_{a}^{u}\right)+g_{i}^{r}\left(\delta_{a}^{u}\right)\right)\left(V_{i a}^{r u}\right)^{2} \\
& +\sum_{u \neq r}^{M} \sum_{a=1}^{n_{u}} \sum_{v=1}^{M} \sum_{b=1}^{n_{v}} c_{i a}^{r u} \beta_{a i b}^{u r v}\left(\frac{S_{i a}^{r u^{*}}}{g_{i}^{r}\left(\delta_{a}^{u}\right)}+\frac{\bar{B}^{2}}{g_{i}^{r}\left(\delta_{a}^{u}\right)}\right)\left(V_{b a}^{v u}\right)^{2}
\end{aligned}
$$

Furthermore, by using Cauchy-Swartz and Hölder inequalities and (4.22), the sixth, seventh and eighth terms in (4.19)-(4.21) are estimated as follows:

$$
\begin{aligned}
& 2 \varrho_{a}^{u} A_{i a}^{r u} \int_{0}^{\infty} V_{i a}^{r u}(t-s) f_{i a}^{r u}(s) e^{-\delta_{a}^{u} s} \mathrm{~d} s \\
& \leq \frac{\left(\varrho_{a}^{u}\right)^{2}}{\mu_{i a}^{r u}} \int_{0}^{\infty}\left(V_{i a}^{r u}(t-s)\right)^{2} f_{i a}^{r u}(s) \mathrm{e}^{-2 \delta_{a}^{u} s} d s+\mu_{i a}^{r u}\left(A_{i a}^{r u}\right)^{2}, \\
& \quad \forall r, u \in I(1, M), i \in I\left(1, n_{r}\right), a \in I\left(1, n_{u}\right), A_{i a}^{r u} \in\left\{U_{i a}^{r u}, V_{i a}^{r u}, W_{i a}^{r u}\right\} .
\end{aligned}
$$


From (4.19)-(4.23), (4.18), repeated usage of (3.25) and inequality (4.22) coupled with some algebraic manipulations and simplifications, we have the following inequality

$$
\begin{aligned}
& L V_{1}\left(\tilde{x}_{00}^{00}\right) \leq \sum_{r=1}^{M} \sum_{i=1}^{n_{r}}\left\{\left[2 \sum_{u=1 a=1}^{M} \sum_{i a}^{n_{u}} \mu_{i a}^{r u}+2 \sum_{a \neq i}^{n_{r}} \frac{\left(\sigma_{i a}^{r r}\right)^{2}}{\mu_{i i}^{r r}}+2 \sum_{u \neq r}^{M} \sum_{a=1}^{n_{r}} \frac{\left(\gamma_{i a}^{r u}\right)^{2}}{\mu_{i i}^{r r}}+4 \mu_{i i}^{r r}-2\left(\gamma_{i}^{r}+\sigma_{i}^{r}+\delta_{i}^{r}\right)\right]\left(U_{i i}^{r r}\right)^{2}\right. \\
& +\left[\left(2+c_{i i}^{r r}\right) \sum_{u=1 a=1}^{M} \sum_{u}^{n_{u}} \mu_{i a}^{r u}+\sum_{a \neq r}^{n_{r}}\left(2+c_{i a}^{r r}\right) \frac{\left(\sigma_{i a}^{r r}\right)^{2}}{\mu_{i i}^{r r}}+\sum_{u=1 a=1}^{M} \sum_{u}^{n_{u}}\left(2+c_{i a}^{r u}\right) \frac{\left(\gamma_{i a}^{r u}\right)^{2}}{\mu_{i i}^{r r}}+\mu_{i i}^{r r}\right. \\
& +\frac{\left(\varrho_{i}^{r}+d_{i}^{r}\right)^{2}}{\mu_{i i}^{r r}}+4 \frac{\left(\gamma_{i}^{r}+\sigma_{i}^{r}+\delta_{i}^{r}\right)^{2}}{\mu_{i i}^{r r}}+\frac{\left(\varrho_{i}^{r}\right)^{2}}{\mu_{i i}^{r r}}+c_{i i}^{r r} \sum_{v=1}^{M} \sum_{b=1}^{n_{r}} \beta_{i i b}^{r r v}\left(S_{i i}^{r *} \mu_{i i}^{r r}+\mu_{i i}^{r r}\right) \\
& \left.-2\left(c_{i i}^{r r}+1\right)\left(\varrho_{i}^{r}+\gamma_{i}^{r}+\sigma_{i}^{r}+\delta_{i}^{r}+d_{i}^{r}\right)\right]\left(V_{i i}^{r r}\right)^{2} \\
& \left.+\left[\sum_{u=1 a=1}^{M} \sum_{i a}^{n_{u}} \mu_{i a}^{r u}+\sum_{a \neq i}^{n_{r}} \frac{\left(\sigma_{i a}^{r r}\right)^{2}}{\mu_{i i}^{r r}}+\sum_{u \neq r a=1 i}^{M} \sum^{n_{r}} \frac{\left(\gamma_{i a}^{r u}\right)^{2}}{\mu_{i i}^{r r}}+2 \mu_{i i}^{r r}-2\left(\gamma_{i}^{r}+\sigma_{i}^{r}+\delta_{i}^{r}\right)\right]\left(W_{i i}^{r r}\right)^{2}\right\} \\
& +\sum_{r=1}^{M} \sum_{i=1}^{n_{r}} \sum_{a \neq i}^{n_{r}}\left\{\left[2 \frac{\left(\rho_{i a}^{r r}\right)^{2}}{\mu_{i a}^{r r}}+2 \mu_{i i}^{r r}+3 \mu_{i a}^{r r}-2\left(\rho_{i a}^{r r}+\delta_{a}^{r}\right)\right]\left(U_{i a}^{r r}\right)^{2}\right. \\
& +\left[\left(2+c_{i i}^{r r}\right) \frac{\left(\rho_{i a}^{r r}\right)^{2}}{\mu_{i a}^{r r}}+\left(2+c_{i a}^{r r}\right) \mu_{i i}^{r r}+\frac{\left(\varrho_{a}^{r}+d_{a}^{r}\right)^{2}}{\mu_{i a}^{r r}}+4 \frac{\left(\rho_{i a}^{r r}+\delta_{a}^{r}\right)^{2}}{\mu_{i a}^{r r}}+\mu_{i a}^{r r}\right. \\
& \left.+c_{i a}^{r r} \sum_{v=1}^{M} \sum_{b=1}^{n_{r}} \beta_{a i b}^{r r v}\left(S_{i a}^{r r *} \mu_{i a}^{r r}+\mu_{i a}^{r r}\right)-2\left(c_{i a}^{r r}+1\right)\left(\varrho_{a}^{r}+\rho_{i a}^{r r}+\delta_{a}^{r}+d_{a}^{r}\right)\right]\left(V_{i a}^{r r}\right)^{2} \\
& \left.+\left[\frac{\left(\rho_{i a}^{r r}\right)^{2}}{\mu_{i a}^{r r}}+\mu_{i i}^{r r}+2 \mu_{i a}^{r r}-2\left(\rho_{i a}^{r r}+\delta_{a}^{r}\right)\right]\left(W_{i a}^{r r}\right)^{2}\right\} \\
& +\sum_{r=1}^{M} \sum_{i=1}^{n_{r}} \sum_{u \neq r a=1}^{M} \sum^{n_{u}}\left\{\left[2 \frac{\left(\rho_{i a}^{r u}\right)^{2}}{\mu_{i a}^{r r}}+2 \mu_{i i}^{r r}+3 \mu_{i a}^{r u}-2\left(\rho_{i a}^{r u}+\delta_{a}^{u}\right)\right]\left(U_{i a}^{r u}\right)^{2}\right. \\
& +\left[\left(2+c_{i i}^{r r}\right) \frac{\left(\rho_{i a}^{r u}\right)^{2}}{\mu_{i a}^{r u}}+\left(2+c_{i a}^{r u}\right) \mu_{i i}^{r r}+\frac{\left(\varrho_{a}^{u}+d_{a}^{u}\right)^{2}}{\mu_{i a}^{r u}}+4 \frac{\left(\rho_{i a}^{r u}+\delta_{a}^{u}\right)^{2}}{\mu_{i a}^{r u}}+\mu_{i a}^{r u}\right. \\
& \left.+c_{i a}^{r u} \sum_{v=1}^{M} \sum_{b=1}^{n_{r}} \beta_{a i b}^{u r v}\left(S_{i a}^{r u^{*}} \mu_{i a}^{r u}+\mu_{i a}^{r u}\right)-2\left(c_{i a}^{r u}+1\right)\left(\varrho_{a}^{u}+\rho_{i a}^{r u}+\delta_{a}^{u}+d_{a}^{u}\right)\right]\left(V_{i a}^{r u}\right)^{2} \\
& \left.+\left[\frac{\left(\rho_{i a}^{r u}\right)^{2}}{\mu_{i a}^{r u}}+\mu_{i i}^{r r}+2 \mu_{i a}^{r u}-2\left(\rho_{i a}^{r u}+\delta_{a}^{u}\right)\right]\left(W_{i a}^{r u}\right)^{2}\right\} \\
& +3 \sum_{r=1}^{M} \sum_{i=1}^{n_{r}} \sum_{u=1}^{M} \sum_{a=1}^{n_{u}} \frac{\left(\varrho_{a}^{u}\right)^{2}}{\mu_{i a}^{r u}} \int_{0}^{\infty}\left(V_{i a}^{r u}(t-s)\right)^{2} f_{i a}^{r u}(s) e^{-2 \delta_{a}^{u} s} \mathrm{~d} s \\
& +\sum_{r=1}^{M} \sum_{i=1}^{n_{r}} c_{i i}^{r r} \sum_{v=1}^{M} \sum_{b=1}^{n_{r}}\left[\beta_{i i b}^{r v}\left(\frac{S_{i i}^{r r *}}{\mu_{i i}^{r r}}+\frac{\bar{B}^{2}}{\mu_{i i}^{r r}}\right)+\left(v_{i i b}^{r v v}\right)^{2}\left(S_{i i}^{r r *}+\bar{B}\right)^{2}\right]\left(V_{b i}^{v r}\right)^{2} \\
& +\sum_{r=1}^{M} \sum_{i=1}^{n_{r}} \sum_{a \neq i}^{n_{r}} c_{i a}^{r r}\left[\sum_{v=1}^{M} \sum_{b=1}^{n_{r}} \beta_{a i b}^{r r v}\left(\frac{S_{i a}^{r r *}}{\mu_{i a}^{r r}}+\frac{\bar{B}^{2}}{\mu_{i a}^{r r}}\right)+\left(v_{a i b}^{r r v}\right)^{2}\left(S_{i a}^{r r *}+\bar{B}\right)^{2}\right]\left(V_{b a}^{v r}\right)^{2} \\
& +\sum_{r=1}^{M} \sum_{i=1}^{n_{r}} \sum_{u \neq r}^{M} \sum_{a=1}^{n_{r}} c_{i a}^{r u}\left[\sum_{v=1}^{M} \sum_{b=1}^{n_{r}} \beta_{a i b}^{u r v}\left(\frac{S_{i a}^{r u^{*}}}{\mu_{i a}^{r u}}+\frac{\bar{B}^{2}}{\mu_{i a}^{r u}}\right)+\left(v_{a i b}^{u r v}\right)^{2}\left(S_{i a}^{r u^{*}}+\bar{B}\right)^{2}\right]\left(V_{b a}^{v u}\right)^{2} \text {, }
\end{aligned}
$$


where $\mu_{i a}^{r u}=g_{i}^{r}\left(\delta_{a}^{u}\right), g_{i}^{r}$ is appropriately defined by (4.22). The differential operator $L V$ [31] [32] applied to the Lyapunov functional (4.10) and (4.11), leads to the following

$$
\begin{aligned}
L V\left(\tilde{x}_{00}^{00}\right) \leq & L V_{1}\left(\tilde{x}_{00}^{00}\right)+3 \sum_{r=1}^{M} \sum_{i=1}^{n_{r}} \sum_{u=1}^{M} \sum_{a=1}^{n_{u}} \frac{\left(\varrho_{a}^{u}\right)^{2}}{\mu_{i a}^{r u}}\left(V_{i a}^{r u}(t)\right)^{2} \int_{0}^{\infty} f_{i a}^{r u}(s) e^{-2 \delta_{a}^{u} s} \mathrm{~d} s \\
& -3 \sum_{r=1}^{M} \sum_{i=1}^{n_{r}} \sum_{u=1}^{M} \sum_{a=1}^{n_{u}} \frac{\left(\varrho_{a}^{u}\right)^{2}}{\mu_{i a}^{r u}} \int_{0}^{\infty}\left(V_{i a}^{r u}(t-s)\right)^{2} f_{i a}^{r u}(s) e^{-2 \delta_{a}^{u} s} \mathrm{~d} s .
\end{aligned}
$$

We note that $\int_{0}^{\infty} f_{i a}^{r u}(s) e^{-2 \delta_{a}^{u} s} \mathrm{~d} s \leq 1$. Furthermore, tt follows from (4.26), (4.25), and some further algebraic manipulations and simplifications that

$$
\begin{aligned}
L V\left(\tilde{X}_{00}^{00}\right) \leq & \sum_{r=1}^{M} \sum_{i=1}^{n_{r}}-\left\{\left[\phi_{i i}^{r r}\left(U_{i i}^{r r}\right)^{2}+\psi_{i i}^{r r}\left(V_{i i}^{r r}\right)^{2}+\varphi_{i i}^{r r}\left(W_{i i}^{r r}\right)^{2}\right]\right. \\
& +\sum_{a \neq r}^{n_{r}}\left[\phi_{i a}^{r r}\left(U_{i a}^{r r}\right)^{2}+\psi_{i a}^{r r}\left(V_{i a}^{r r}\right)^{2}+\varphi_{i a}^{r r}\left(W_{i a}^{r r}\right)^{2}\right] \\
& \left.+\sum_{u \neq r a=1}^{M} \sum_{n_{u}}^{n_{u}}\left[\phi_{i a}^{r u}\left(U_{i a}^{r u}\right)^{2}+\psi_{i a}^{r u}\left(V_{i a}^{r u}\right)^{2}+\varphi_{i a}^{r u}\left(W_{i a}^{r u}\right)^{2}\right]\right\} .
\end{aligned}
$$

where, for each $r, u \in I(1, M), \quad i \in I\left(1, n_{r}\right)$ and $a \in I\left(1, n_{u}\right)$, using (4.12), (4.13) and (4.14), we define the constants $d_{a i}^{u r}, \phi_{i a}^{r u}, \psi_{i a}^{r u}$ and $\varphi_{i a}^{r u}$ as follows:

$$
d_{a i}^{u r}=\sum_{v=1}^{M} \sum_{b=1}^{n_{v}} c_{b a}^{v u} \beta_{a b i}^{u v r}\left(\frac{S_{b a}^{v u^{*}}+\bar{B}^{2}}{\mu_{b a}^{v u}}\right)+\sum_{v=1}^{M} \sum_{b=1}^{n_{v}} c_{b a}^{v u}\left(v_{a b i}^{u v r}\right)^{2}\left(S_{b a}^{v u^{*}}+\bar{B}\right)^{2}
$$

for some positive numbers $c_{i a}^{r u}$, for all $r, u \in I^{r}(1, M), \quad i \in I(1, n)$ and $a \in I_{i}^{r}\left(1, n_{r}\right)$.

$$
\begin{gathered}
\phi_{i a}^{r u}=\left\{\begin{array}{l}
2\left(\gamma_{i}^{r}+\sigma_{i}^{r}+\delta_{i}^{r}\right)\left(1-\mathfrak{U}_{i a}^{r u}\right), \text { for } u=r, a=i \\
2\left(\rho_{i a}^{r r}+\delta_{a}^{r}\right)\left(1-\mathfrak{U}_{i a}^{r u}\right), \text { for } u=r, a \neq i \\
2\left(\rho_{i a}^{r u}+\delta_{a}^{u}\right)\left(1-\mathfrak{U}_{i a}^{r u}\right), \text { for } u \neq r,
\end{array}\right. \\
\psi_{i a}^{r u}=\left\{\begin{array}{l}
2\left(\varrho_{i}^{r}+\gamma_{i}^{r}+\sigma_{i}^{r}+\delta_{i}^{r}+d_{i}^{r}\right)\left[c_{i i}^{r r}\left(1-\mathfrak{V}_{i i}^{r r}\right)+\left(1-\frac{1}{2} \mathfrak{E}_{i i}^{r r}\right)\right], \text { for } u=r, a=i \\
2\left(\varrho_{a}^{r}+\rho_{i a}^{r r}+\delta_{a}^{r}+d_{a}^{r}\right)\left[c_{i a}^{r r}\left(1-\mathfrak{V}_{i a}^{r r}\right)+\left(1-\frac{1}{2} \mathfrak{E}_{i a}^{r r}\right)\right], \text { for } u=r, a \neq i \\
2\left(\varrho_{a}^{u}+\rho_{i a}^{r u}+\delta_{a}^{u}+d_{a}^{u}\right)\left[c_{i a}^{r u}\left(1-\mathfrak{V}_{i a}^{r u}\right)+\left(1-\frac{1}{2} \mathfrak{E}_{i a}^{r u}\right)\right], \text { for } u \neq r
\end{array}\right.
\end{gathered}
$$

and

$$
\varphi_{i a}^{r u}=\left\{\begin{array}{l}
2\left(\gamma_{i}^{r}+\sigma_{i}^{r}+\delta_{i}^{r}\right)\left(1-\mathfrak{W}_{i a}^{r u}\right), \text { for } u=r, a=i, \\
2\left(\rho_{i a}^{r r}+\delta_{a}^{r}\right)\left(1-\mathfrak{W}_{i a}^{r u}\right), \text { for } u=r, a \neq i, \\
2\left(\rho_{i a}^{r u}+\delta_{a}^{u}\right)\left(1-\mathfrak{W}_{i a}^{r u}\right), \text { for } u \neq r
\end{array}\right.
$$

moreover, $\mathfrak{U}_{i a}^{r u}, \mathfrak{V}_{i a}^{r u}, \mathfrak{W}_{i a}^{r u}$ are given in (4.12), (4.13), (4.14) and 


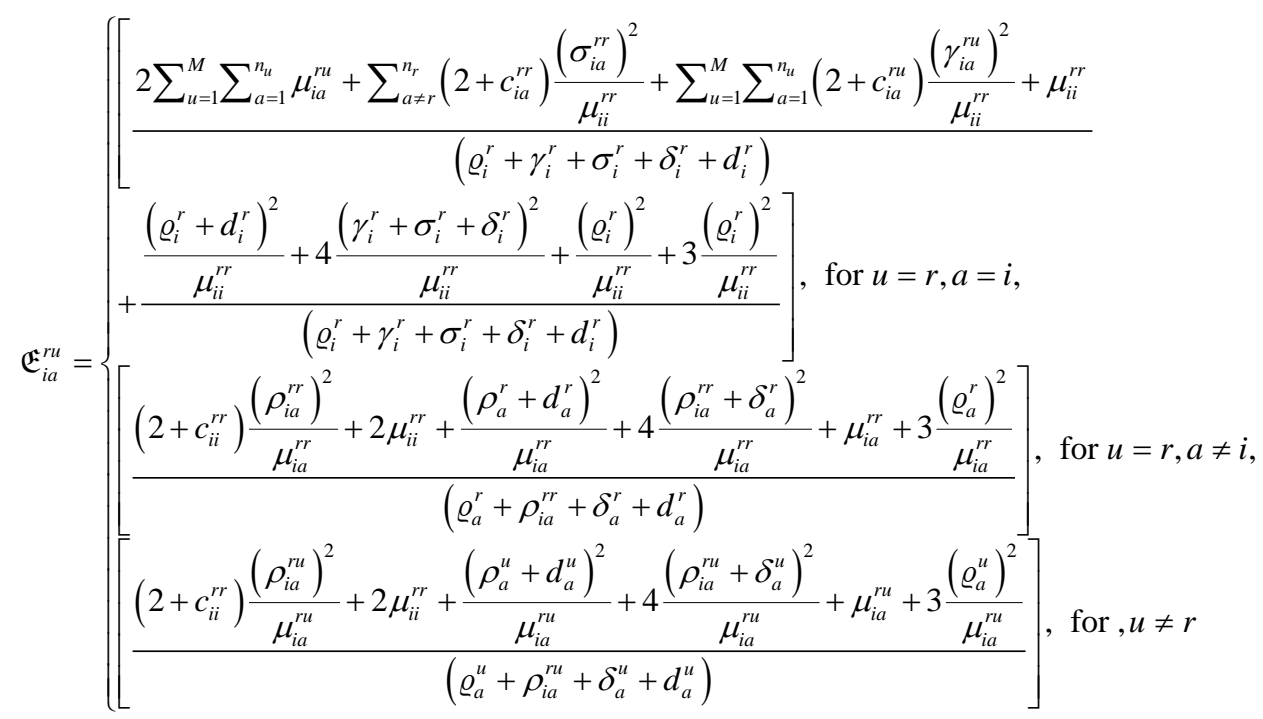

Under the assumptions on $\mathfrak{U}_{i a}^{r u}, \mathfrak{V}_{i a}^{r u}$ and $\mathfrak{W}_{i a}^{r u}$, it is clear that $\phi_{i a}^{r u}, \psi_{i a}^{r u}$ and $\varphi_{i a}^{r u}$ are positive for suitable choices of the constants $c_{i a}^{r u}>0$. Thus this proves the inequality (4.15). Now, the validity of (4.16) follows from (4.15) and (4.8), that is,

$$
L V\left(\tilde{x}_{00}^{00}\right) \leq-c V_{1}\left(\tilde{x}_{00}^{00}\right),
$$

where $c=\frac{\min _{1 \leq r, u \leq M, 1 \leq i \leq n_{r}, 1 \leq a \leq n_{u}}\left\{\phi_{i a}^{r u}, \psi_{i a}^{r u}, \varphi_{i a}^{r u}\right\}}{\max _{1 \leq r, u \leq M, 1 \leq i \leq n_{r}, 1 \leq a \leq n_{u}}\left\{C_{i a}^{r u}+2\right\}}$. This completes the proof. We now formally state the stochastic stability theorems for the disease free equilibria.

Theorem 4.1. Given $r, u \in I(1, M), i \in I\left(1, n_{r}\right)$ and $a \in I\left(1, n_{u}\right)$. Let us assume that the hypotheses of Lemma 4.2 are satisfied. Then the disease free solutions $E_{i a}^{r u}$, are asymptotically stable in the large. Moreover, the solutions $E_{i a}^{r u}$ are exponentially mean square stable.

Proof:

From the application of comparison result [31] [32], the proof of stochastic asymptotic stability follows immediately. Moreover, the disease free equilibrium state is exponentially mean square stable. We now consider the following corollary to Theorem 4.1.

Corollary 4.1. Let $r \in I(1, M)$ and $i \in I\left(1, n_{r}\right)$. Assume that $\sigma_{i}^{r}=\gamma_{i}^{r}=0$, for all $r \in I(1, M)$ and $i \in I\left(1, n_{r}\right)$.

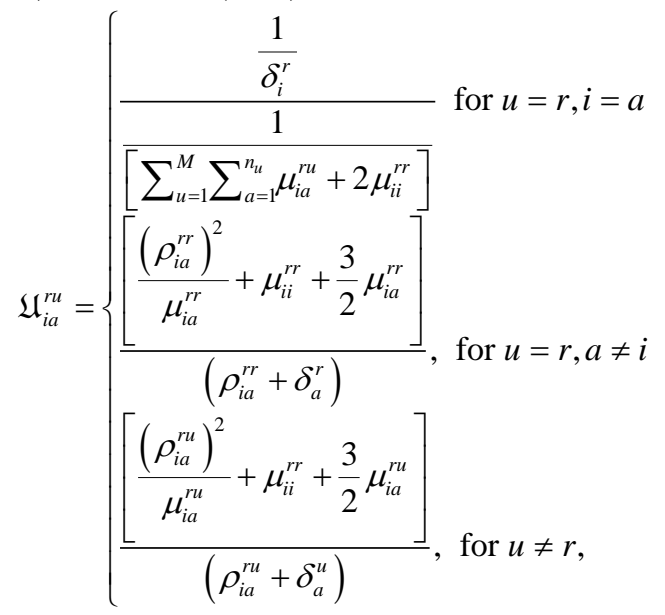




$$
\mathfrak{V}_{i a}^{r u}=\left\{\begin{array}{l}
\frac{\frac{1}{2} \sum_{u=1}^{M} \sum_{a=1}^{n_{u}} \mu_{i a}^{r u}+\frac{1}{2} \sum_{v=1}^{M} \sum_{b=1}^{n_{v}} \beta_{i i b}^{r r v}\left(S_{i i}^{r r *} \mu_{i i}^{r r}+\mu_{i i}^{r r}\right)+\frac{1}{2} d_{i i}^{r r}}{\varrho_{i}^{r}+\delta_{i}^{r}+d_{i}^{r}}, \text { for } a=i, u=r \\
\frac{\frac{1}{2} \mu_{i i}^{r r}+\frac{1}{2} \sum_{v=1}^{M} \sum_{b=1}^{n_{v}} \beta_{a i b}^{r r v}\left(S_{i a}^{r r *} \mu_{i a}^{r r}+\mu_{i a}^{r r}\right)+\frac{1}{2} d_{a i}^{r r}}{\varrho_{a}^{r}+\rho_{i a}^{r r}+\delta_{a}^{r}+d_{a}^{r}}, \text { for } a \neq i, u=r \\
\frac{\frac{1}{2} \mu_{i i}^{r r}+\frac{1}{2} \sum_{v=1}^{M} \sum_{b=1}^{n_{v}} \beta_{a i b}^{u r v}\left(S_{i i}^{r u^{*}} \mu_{i a}^{r u}+\mu_{i a}^{r u}\right)+\frac{1}{2} d_{a i}^{u r}}{\varrho_{a}^{u}+\rho_{i a}^{r u}+\delta_{a}^{u}+d_{a}^{u}}, \text { for } u \neq r .
\end{array}\right.
$$

and

$$
\mathfrak{W}_{i a}^{r u}=\left\{\begin{array}{l}
\frac{\frac{1}{\delta_{i}^{r}}}{\frac{1}{\left[\frac{1}{2} \sum_{u=1}^{M} \sum_{a=1}^{n_{u}} \mu_{i a}^{r u}+\mu_{i i}^{r r}\right]}}, \text { for } u=r, a=i, \\
\frac{\left.\frac{1}{2} \frac{\left(\rho_{i a}^{r r}\right)^{2}}{\mu_{i a}^{r r}}+\frac{1}{2} \mu_{i i}^{r r}+\mu_{i a}^{r r}\right]}{\left(\rho_{i a}^{r r}+\delta_{a}^{r}\right)}, \text { for } u=r, a \neq i, \\
\frac{\left[\frac{1}{2} \frac{\left(\rho_{i a}^{r u}\right)^{2}}{\mu_{i a}^{r u}}+\frac{1}{2} \mu_{i i}^{r r}+\mu_{i a}^{r u}\right]}{\left(\rho_{i a}^{r u}+\delta_{a}^{u}\right)}, \text { for } u \neq r
\end{array}\right.
$$

The equilibrium state $E_{i i}^{r r}$ is stochastically asymptotically stable provided that $\mathfrak{U}_{i a}^{r u}, \mathfrak{W}_{i a}^{r u} \leq 1$ and $\mathfrak{V}_{i a}^{r u}<1$, for all $u \in I^{r}(1, M)$ and $a \in I_{i}^{r}\left(1, n_{u}\right)$.

Proof: Follows immediately from the hypotheses of Lemma 4.2, (letting $\sigma_{i}^{r}=\gamma_{i}^{r}=0$ ), the conclusion of Theorem 4.1 and some algebraic manipulations.

Remark 4.2.

1. The presented results about the two-level large scale delayed SIR disease dynamic model depend on the underlying system parameters. In particular, the sufficient conditions are algebraically simple, computationally attractive and explicit in terms of the rate parameters. As a result of this, several scenarios can be discussed and exhibit practical course of action to control the disease. For simplicity, we present an illustration as follows: the conditions of $\sigma_{i}^{r}=\gamma_{i}^{r}=0$, $\forall r, i$ in Corollary 4.1 signify that the arbitrary site $s_{i}^{r}$ is a "sink" in the context of compartmental systems [33] [34] for all other sites in the inter and intraregional accessible domain. This scenario is displayed in Figure 1. The conditions $\mathfrak{U}_{i i}^{r r} \leq 1$ and $\mathfrak{W}_{i i}^{r r} \leq 1$ exhibit that the average life span is smaller than the joint average life span of individuals in the intra and inter-regional accessible domain of site $s_{i}^{r}$. Furthermore, the conditions $\mathfrak{V}_{i a}^{r u}<1, \forall u \in I(1, M), a \in I\left(1, n_{r}\right)$, and $\mathfrak{U}_{i a}^{r u} \leq 1, \mathfrak{W}_{i a}^{r u} \leq 1, \forall u=r, a \neq i$, and $\forall u \neq r, a \in I\left(1, n_{r}\right)$, signify that the magnitude of disease inhibitory processes for example, the magnitude of the recovery process is greater than the disease transmission process. A future detailed study of the disease dynamics in the two scale network dynamic 


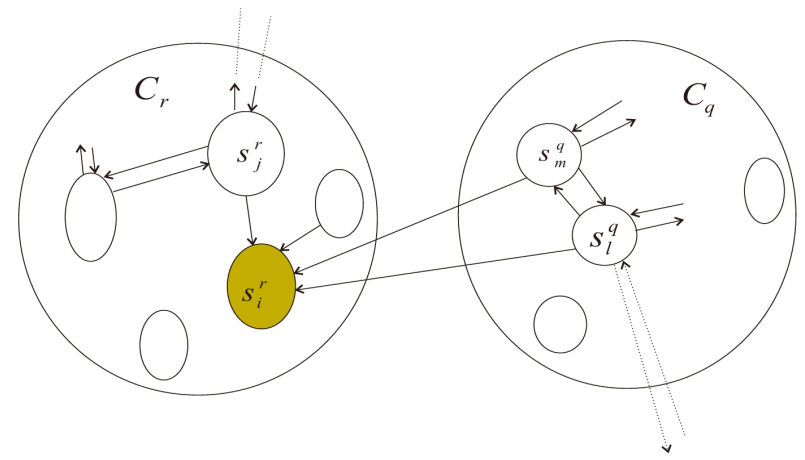

Figure 1. Shows that residents of site $s_{i}^{r}$ are present only at their home site $s_{i}^{r}$. Hence they isolate every site from their inter and intra regional accessible domain $C\left(s_{i}^{r}\right)$. Site $s_{i}^{r}$ is a "sink" in the context of the compartmental system [33] [34]. The arrows represent a transport network between any two sites and regions. Furthermore, the dotted lines and arrows indicate connection with other sites and regions.

structure for many real life scenarios using the presented two level large-scale delay SIR disease dynamic model will appear elsewhere.

2. The stochastic delayed epidemic model (2.1)-(2.3) is a general representation of infection acquired immunity delay in a two-scale network population disease dynamics. The stochastic delayed epidemic model with temporary immunity period ((2.7)-(2.9), [22]) and the numerical simulation results (Section 5, [22]) are special cases of (2.1)-(2.3) when we let the probability density function of the immunity period, $f_{i a}^{r u}(s)=\delta\left(s-T_{i}^{r}\right), \quad \forall r, u \in I(1,3)$, $\forall i, a \in I(1,3)$, where $\delta$ is the Dirac $\delta$-function [19].

\section{Conclusions}

The presented two-scale network delayed epidemic dynamic model with varying immunity period characterizes the dynamics of an SIR epidemic in a population with various scale levels created by the heterogeneities in the population. Moreover, the disease dynamics is subject to random environmental perturbations at the disease transmission stage of the disease. Furthermore, the SIR epidemic confers varying time temporary acquired immunity to recovered individuals immediately after recovery. This work provides a mathematical and probabilistic algorithmic tool to develop different levels nested type disease transmission rates, the variability in the transmission process as well as the distributed time delay in the framework of the network-centric Ito-Doob type dynamic equations. In addition, the concept of distributed delay caused by the acquired immunity period in the dynamics of human epidemics is explored for the first time in the context of complex scale-structured type human metapopulations.

The model validation results are developed and a positively self invariant set for the dynamic model is defined. Moreover, the globalization of the positive solution existence is obtained by applying an energy function method. In addition, using the Lyapunov functional technique, the detailed stochastic asym- 
ptotic stability results of the disease free equilibria are also exhibited in this paper. Moreover, the system parameter values dependent threshold values controlling the stochastic asymptotic stability of the disease free equilibrium are also defined. Furthermore, a deduction to the stochastic asymptotic stability results for a simple real life scenario is illustrated. We note, further detail study of the stochastic SIR human epidemic dynamic model with varying immunity period for two scale network mobile population exhibiting several real life human mobility patterns will appear elsewhere.

We note that the disease dynamics is subject to random environmental perturbations from other related sub-processes such as the mobility, recovery, birth and death processes. The variability due to the disease transmission incorporated in the epidemic dynamic model will be extended to the variability in the mobility, recovery and birth and death processes. A further detailed study of the oscillation of the epidemic process about the ideal endemic equilibrium of the dynamic epidemic model will also appear else where. In addition, a detailed study of the hereditary features of the infectious agent such as the time-lag to infectiousness of exposed individuals in the population is currently underway and it will also appear elsewhere.

\section{Acknowledgements}

This research was supported by the Mathematical Science Division, US Army Research Office, Grant No. W911NF-12-1-0090.

\section{References}

[1] Knobler, S., Mahmoud, A., Lemon, S. and Pray, L. (2006) The Impact of Globalization on Infectious Disease Emergence and Control: Exploring the Consequences and Opportunities, Workshop Summary-Forum on Microbial Threats. The National Academies Press, Washington DC.

[2] 2009 H1N1 Flu: International Situation Update. http://www.cdc.gov/h1n1flu/updates/international/

[3] Rvachev, L. and Longini, I. (1985) A Mathematical Model for the Global Spread of Influenza. Mathematical Biosciences, 75, 3-22.

[4] Longini, I. (1988) A Mathematical Model for Predicting the Geographic Spread of New Infectious Agents. Mathematical Biosciences, 90, 367-383.

[5] Wang, W. and Zhao, X.-Q. (2004) An Epidemic Model in a Patchy Environment. Mathematical Biosciences, 190, 97-112.

[6] Wang, W. and Zhao, X.-Q. (2005) An Age-Structured Epidemic Model in a Patchy Environment. SIAM Journal on Applied Mathematics, 65, 1597-1614. https://doi.org/10.1137/S0036139903431245

[7] Cosner, C., Beier, J.C., Cantrell, R.S., Impoinvil, D., Kapitanski, L., Potts, M.D., Troyo, A. and Ruan, S. (2009) The Effects of Human Movement on the Persistence of Vector Borne Diseases. Journal of Theoretical Biology, 258, 550-560.

[8] Arino, J. and Van den Driessche, P. (2003) A Multi-City Epidemic Model. Mathematical Population Studies, 10, 175-193. https://doi.org/10.1080/08898480306720

[9] Arino, J., Davis, J.R., Hartley, D., Jordan, R., Miller, J.M. and van den Driessche, P. (2005) A Multi-Species Epidemic Model with Spatial Dynamics. Mathematical 
Medicine and Biology, 22, 129-142. https://doi.org/10.1093/imammb/dqi003

[10] Sattenspiel, L. and Dietz, K. (1995) A Structured Epidemic Model Incorporating Geographic Mobility among Regions. Mathematical Biosciences, 128, 7191.

[11] Sattenspiel, L. and Herring, D.A. (1998) Structured Epidemic Models and the Spread of Influenza in the Central Canada Subarctic. Human Biology, 70, 91-115.

[12] Sattenspiel, L. and Herring, D.A. (2003) Simulating the Effect of Quarantine on the Spread of the 1918-1919 Flu in Central Canada. Bulletin of Mathematical Biology, 65, 1-26. https://doi.org/10.1006/bulm.2002.0317

[13] Jin, Y., Wang, W. and Xiao, S. (2007) An SIRS Model with a Nonlinear Incidence Rate. Chaos, Solitons Fractals, 34, 1482-1497.

[14] Ruan, S., Wang, W. and Levin, S.A., et al. (2006) The Effect of Global Travel on the Spread of SARS. Mathematical Biosciences and Engineering, 3, 205-218. https://doi.org/10.3934/mbe.2006.3.205

[15] Rodriguez, D.J. and Torres-Sorando, L. (2001) Models for Infectious Diseases in Spatially Heterogeneous Environments. Bulletin of Mathematical Biology, 63, 547571. https://doi.org/10.1006/bulm.2001.0231

[16] Beretta, E., Hara, T., Ma, W. and Takeuchi, Y. (2001) Global Asymptotic Stability of an SIR Epidemic Model with Distributed Time Delay. Nonlinear Analysis, 47, 4107 4115.

[17] Gao, S., Teng, Z., Nieto, J.J. and Torres, A. (2007) Analysis of an SIR Epidemic Model with Pulse Vaccination and Distributed Time Delay. Journal of Biomedicine and Biotechnology, 2007, Article ID: 64870. https://doi.org/10.1155/2007/64870

[18] Arino, J., Cooke, K.L., van den Driessche, P. and Velasco-Herndez, J. (2004) An Epidemiology Model That Includes a Leaky Vaccine with a General Waining Function. Discrete and Continuous Dynamical Systems, 2, 479-495.

[19] Blyuss, K.B. and Kyrychko, Y.N. (2010) Stability and Bifurcations in an Epidemic Model with Varying Immunity Period. Bulletin of Mathematical Biology, 72, 490505. https://doi.org/10.1007/s11538-009-9458-y

[20] Tornatore, E., Buccellato, S.M. and Vetro, P. (2005) Stability of a Stochastic SIR System. Journal of Physics A, 354, 111-126.

[21] Wanduku, D. and Ladde, G.S. (2011) Fundamental Properties of a Two-Scale Network Stochastic Human Epidemic Dynamic Model. Neural, Parallel, and Scientific Computations, 19, 229-270.

[22] Wanduku, D. and Ladde, G.S. (2012) Global Properties of a Two-Scale Network Stochastic Delayed Human Epidemic Dynamic Model. Nonlinear Analysis. Real World Applications, 13, 794-816.

[23] Chen, G. and Li, T. (2009) Stability of Stochastic Delayed SIR Model. Stochastics and Dynamics, 9, 231-252. https://doi.org/10.1142/S0219493709002658

[24] Gray, A., Greenhalgh, D., Hu, L., Mao, X. and Pan, J. (2011) A Stochastic Differential Equation SIS Epidemic Model. SIAM Journal on Applied Mathematics, 71, 876902. https://doi.org/10.1137/10081856X

[25] Lu, Q. (2009) Stability of SIRS System with Random Perturbations. Physica A, 338, 3677-3686.

[26] Lahrouz, A., Omari, L. and Kiouach, D. (2011) Global Analysis of a Deterministic and Stochastic Nonlinear SIRS Epidemic Model. Nonlinear Analysis: Modelling and Control, 16, 59-76.

[27] Dalala, N., Greenhalgh, D. and Mao, X. (2007) A Stochastic Model of AIDS and Condom Use. Journal of Mathematical Analysis and Applications, 325, 36-53. 
[28] Dalala, N., Greenhalgh, D. and Mao, X. (2008) A Stochastic Model for Internal HIV Dynamics. Journal of Mathematical Analysis and Applications, 341, 1084-1101.

[29] Yu, J., Jiang, D. and Shi, N. (2009) Global Stability of Two-Group SIR Model with Random Perturbation. Journal of Mathematical Analysis and Applications, 360, 235-244.

[30] Wanduku, D. and Ladde, G.S. (2010) A Two-Scale Network Dynamic Model for Human Mobility Process. Mathematical Biosciences, 229, 1-15.

[31] Mao, X. (2008) Stochastic Differential Equations and Applications. 2nd Edition, Horwood Publishing Ltd., Sawston. https://doi.org/10.1533/9780857099402

[32] Ladde, G.S. and Lakshmikantham, V. (1980) Random Differential Inequalities. Academic Press, New York.

[33] Ladde, G.S. (1976) Cellular Systems-II. Stability of Campartmental Systems. Mathematical Biosciences, 30, 1-21.

[34] Ladde, G.S. (1976) Cellular Systems-I. Stability of Chemical Systems. Mathematical Biosciences, 29, 309-330.

Submit or recommend next manuscript to SCIRP and we will provide best service for you:

Accepting pre-submission inquiries through Email, Facebook, LinkedIn, Twitter, etc. A wide selection of journals (inclusive of 9 subjects, more than 200 journals)

Providing 24-hour high-quality service

User-friendly online submission system

Fair and swift peer-review system

Efficient typesetting and proofreading procedure

Display of the result of downloads and visits, as well as the number of cited articles

Maximum dissemination of your research work

Submit your manuscript at: http://papersubmission.scirp.org/

Or contact jamp@scirp.org 